\title{
Earthquake Precursory Effects Due to Pore Fluid Stabilization of a Weakening Fault Zone
}

\author{
JAMES R. RICE \\ Division of Engineering, Brown University, Providence, Rhode Island 02912 \\ JOHN W. RUDNICKI ${ }^{1}$ \\ Seismological Laboratory, California Institute of Technology. Pasadena, California 91125

\begin{abstract}
We report the analysis of two mechanisms by which pore fluids could partially stabilize the earthquake rupture process in natural rock masses. These mechanisms are based on dilatancy strengthening and on the increase of elastic stiffness for undrained as opposed to drained conditions. Both are studied in relation to an inclusion model in which a zone of strain weakening material, possibly representing a highly stressed seismic gap zone, is embedded in nominally elastic surroundings subjected to steadily increasing tectonic stress. Owing to the coupling between deformation and pore fluid diffusion, the inclusion does not exhibit an abrupt rupture instability; rather, a period of self-driven precursory creep occurs which ultimately accelerates to dynamic instability. The precursory time scale is reported for a wide range of constitutive parameters, including fluid diffusivity, ratio of undrained to drained stiffness, and factors expressive of strain softening and dilatancy. Our conclusions are that the precursory times for a spherical inclusion of $1-\mathrm{km}$ radius are of the order of 15-240 days for a range of constitutive parameters that we suggest are representative. The predicted times are shorter by a factor of approximately 10 for a flattened ellipsoidal inclusion that we analyze with an 18:1 aspect ratio. It is suggested that perhaps only toward the latter part of the precursory period are the effects of accelerating inclusion strain detectable in terms of surface deformation or alteration of transport or seismic properties.
\end{abstract}

\section{INTRODUCTION}

We consider the possibility that rock near sites of shallow earthquakes is infiltrated with groundwater and examine quantitatively processes by which mechanical coupling of rock deformation and pore fluid diffusion could transiently stabilize the rock against rapid failure. The stabilization results from dilatant strengthening within a fault region and from timedependent relaxation of the effective elastic unloading stiffness of the surroundings from undrained to drained conditions. Both mechanisms are shown to allow failure to occur in a less abrupt manner than was predicted without consideration of fluid coupling. Instead of an instantaneous dynamic instability, there is a period of initially quasi-static deformation that proceeds on a time scale governed by pore fluid diffusion and nonelastic deformation characteristics of the failing material and that ultimately accelerates to dynamic instability.

The time scale and character of this quasi-stable deformation has interest as a possible basis for discernible precursors to earthquakes, in the form of accelerating strain or tilt and local pore pressure alterations. Our aim in the present work is to develop quantitative estimates of these precursory processes on the basis of mechanically consistent models of the failure process. In doing so, we do not, of course, claim that all timedependent precursory phenomena are traceable to such mechanical effects of pore fluids in an essentially deformationrate-insensitive rock skeleton. Indeed, some precursory effects arise inevitably from the amplification of local fault region strain rates over remote tectonic strain rates as instability conditions are approached [e.g., Rice, 1977a]. Also, time dependence from corrosive microcracking of stressed rock, creep processes at depth, time-dependent adherence at frictional

\footnotetext{
${ }^{1}$ Now at the Department of Theoretical and Applied Mechanics, University of Illinois, Urbana, Illine: 61801 .
}

Copyright (C) 1979 by the American Geophysical Union.

contacts, and perhaps other mechanisms could be important in different settings.

The first mechanism of fluid coupling that we consider, namely, dilatant hardening, is well known in the mechanics of granular materials [Reynolds, 1885]. Because rock masses and fault zones typically dilate when deformed inelastically, the local pore fluid pressure will decrease unless the deformation is sufficiently slow to permit alleviation of the induced suctions by diffusion. This decrease in pore fluid pressure causes the effective stress (that is, the total stress minus the pore fluid pressure) to increase in compression, thereby inhibiting inelastic deformation mechanisms such as frictional sliding and microcracking. The relevance of dilatant hardening to seismic processes was first suggested by Frank [1965], and it has been studied in the laboratory deformation of rock by Brace and Martin [1968]. A preliminary analysis of the role of dilatancy in stabilization of faulting, in the spirit of that to be discussed here, has been given by Rudnicki [1977b].

The second mechanism by which the interaction of deformation and pore fluid diffusion can stabilize against rapid failure arises because of the dependence of fault instability conditions on the effective elastic stiffness of the fault's surroundings. Such stiffness dependencies are well known in laboratory studies of failure [Jaeger and Cook, 1976]; the stiffer the loading apparatus, the more rock can be deformed stably into the postpeak range. For fluid-infiltrated rock the surroundings are elastically stiffer for stress alterations which are rapid in comparison to diffusive relaxation times than for long-term stress alterations. If the time scale of deformation is rapid in comparison to that for diffusion so that the fluid mass in material elements remains constant, the response is termed 'undrained' and is elastically stiffer than the long-time or 'drained' response for which the local pore fluid pressure is constant. This time dependence provides a mechanism of transient stabilization as discussed by Rice et al. [1978] and Rice [1977a] on the basis of analyses using the linear elastic theory of Biot [1941] 


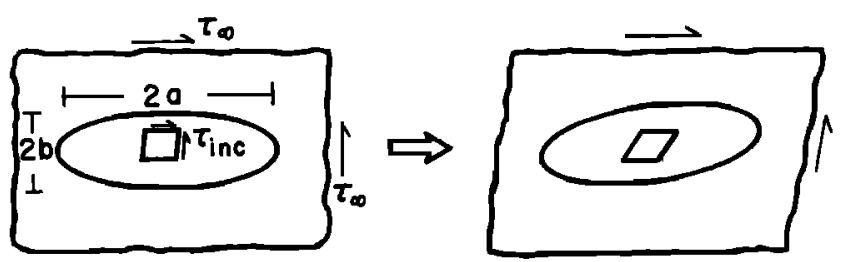

(a)
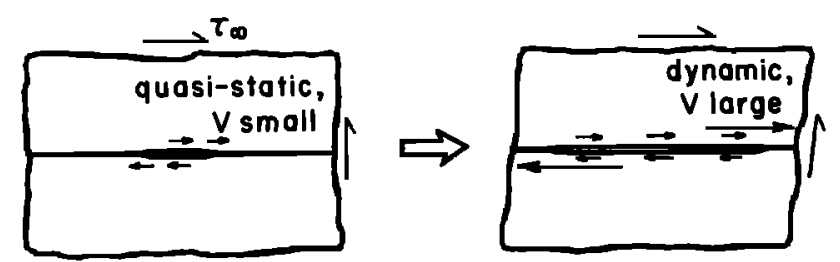

(b)

Fig. 1. Types of failure models. (a) Deformation of 'inclusion' of different mechanical properties. Runaway instability based on strain softening of inclusion and elastic unloading stiffness. (b) Isolated region of slippage on preexisting fault, spreading quasi-statically at small speed $V$.

for porous solids. Similar effects have been studied in problems related to aftershock mechanisms by Booker [1974].

Our analysis of both of the foregoing pore fluid coupling effects is based on a model for the inception of faulting introduced by Rudnicki [1977a]. This model considers the deformation of a rock mass which contains an ellipsoidal inclusion in which the properties are uniform but different from those of the surroundings (Figure 1a). Rudnicki [1977a] interpreted this inclusion as deforming nonlinearly and exhibiting a peak stress as a consequence of weakening by faulting, whereas the surrounding material remains nominally elastic. Rice [1977a], however, has indicated that the model applies as well and, indeed, may be more pertinent if the inclusion is interpreted as sustaining higher stresses than its surroundings. This would be the case if the inclusion had undergone less strain due to past faulting than the surroundings and is, in effect, a 'seismic gap' zone. Because of the large local stress, the inclusion [Rudnicki, $1977 a]$ exhibits a peak stress, whereas the surrounding material remains elastic.

In either interpretation the slow increase of tectonic stress drives the inclusion material past peak stress. At some point the slope of the descending stress-strain curve for the inclusion material becomes sufficiently negative so that no further increase of tectonic stress can be accommodated quasi-statically. This dynamic 'runaway' is interpreted as the occurrence of an earthquake. As we shall see, and as was suggested by prior discussions of the inclusion model by Rudnicki $[1977 a, b]$, Rice et al. [1978], and Rice [1977a], the effect of fluid coupling mechanisms of the type discussed earlier is to cause this instability to occur not abruptly but rather in a more gradual manner with a time scale controlled by fluid diffusion, and, possibly, with discernible precursors.

Despite the simplicity of this model it is motivated by the idea that prefailure processes are likely to be dominated by large-scale heterogeneities of mechanical properties which remain as a result of the past history of faulting. Indeed, because the material properties and the geometry of such fault zones are at present so poorly known, it seems unwise to concoct overly detailed models, although we do believe that simple analyses founded upon consistent mechanical principles are useful for identifying the salient features of precursory processes.

Although our discussion here is organized primarily with reference to the inclusion model, another model which is relevant and may be more appropriate in many circumstances is one in which nonelastic deformations are assumed to be localized along a narrow fault from the outset and in which the slipping zone along the fault can propagate in a shear-cracklike mode (Figure $1 b$ ). In the presence of an infiltrating pore fluid, both the stabilizing effects of dilatant hardening and of time-dependent stiffness of the surroundings are applicable here as well, as has been remarked by Rice and Cleary [1976]. Rice and Simons [1976] examined further the stabilization which results from time-dependent elasticity by solving for the stress concentration near the tip of a quasi-statically propagating shear crack in a fluid-infiltrated porous elastic solid. They found that for a range of crack speeds comparable to propagation rates inferred for episodic creep events in central California [King et al., 1973], the crack extended stably in the sense that with increasing propagation speed an increase in far-field driving stress, relative to frictional resistance, was needed to maintain the same stress intensity near the tip. Also, by adapting an earlier.analysis developed for slip surface propagation in overconsolidated clay soils, Rice [1977a] developed an expression for the increase in far-field driving stress that is required, with increasing propagation speed, to overcome the augmented frictional resistance due to dilatantly induced suctions from the shear 'breakdown' process near a fault tip. The result suggests that the maximum induced suction and required driving stress increase approximately in proportion to $V^{1 / 2}$ at low speeds, where $V$ is the propagation speed. These studies of pore fluid effects on the criterion for propagation of a shear crack suggest precursory effects similar to those that we discuss here on the basis of the inclusion model. In the inclusion model as we present it, an entire zone deforms into the inelastic range and ultimately becomes unstable. The shear crack model is more complicated because it involves the gradual enlargement of the nonelastic (slipping) region. Fluid effects of the kind discussed above provide a possible mechanism for control of the time scale and extent of enlargement of the slip region before an unstable, dynamic spreading of the slip zone occurs, just as the effects may control the time scale and extent of nonelastic strain in the inclusion model. We leave as a goal for future work the quantification of the precursory time scale predicted on the basis of the shear crack model and concentrate here on the inclusion model.

In addition to the mechanical effects of the pore fluid, there are also surface chemical effects. These seem to be important in time-dependent crack growth in quartz-based rocks [e.g., Martin, 1972; Scholz, 1972; Swolfs, 1972; Martin and Durham, $1975 ; O$. L. Anderson and P. C. Grew, 1977], and they may be significant in determining the strength of rocks on a time scale comparable to that for tectonic alterations in stress. The constitutive description of these effects, however, is as yet insufficient to permit incorporation into the rupture models discussed here. Their presence, as well as the presence of time dependence of the frictional resistance of rock [Dieterich. 1972, 1978], would not invalidate the mechanical effects of pore fluids that we discuss here but would add additional components to the overall precursory time-dependent deformation. 
We will begin by reviewing in more detail the inclusion model for instability based on the deformation of an inhomogeneous zone. Then we will analyze the stabilizing effects of the pore fluid by employing the solution of Rice et al. [1978], discussing dilatant hardening on the basis of the work of Rudnicki [1977b] and time-dependent stiffness on the basis of the work of Rice [1977a]. In particular, we will demonstrate that both the mechanisms of dilatant hardening of the inclusion material and time dependence of elastic stiffness of the surrounding material can stabilize the rock mass against dynamic failure at a point where runaway would occur if the infiltrating fluid were not present. The subsequent deformation is initially slow but is self-driving and ultimately accelerates to dynamic instability. Because this self-driven accelerating deformation may display itself in observable precursors, such as accelerated creep or anomalous tilting of the ground surface, we estimate its time duration on the basis of the models presented here and the incorporation of plausible material properties.

\section{INSTABILITY OF A Rock Mass With AN INHOMOGENEOUS ZONE}

As discussed in the introduction, we consider a rock mass containing an inclusion in which the mechanical properties differ from those of the surroundings (Figure 1a), and for convenience of analysis we assume that the inclusion is ellipsoidal in shape. For the present we neglect pore fluid effects and consider the material surrounding the inclusion to be linearly elastic. If, in addition, the inclusion material is homogeneous, the inclusion deforms homogeneously [Eshelby, 1957] even if its material is nonlinear. As a result the difference between the uniform strain of the inclusion $\left(\epsilon_{i j}\right)_{1 \mathrm{nc}}$ and that applied in the far field $\left(\epsilon_{i j}\right)_{\infty}$ is related linearly to the corresponding difference in stresses:

$$
\left(\epsilon_{l j}\right)_{1 \mathrm{nc}}-\left(\epsilon_{l l}\right)_{\infty}=Q_{l j k l}\left[\left(\sigma_{k l}\right)_{\infty}-\left(\sigma_{k l}\right)_{I \mathrm{nc}}\right]
$$

where $\left(\epsilon_{l}\right)_{\infty}$ is related to $\left(\sigma_{l j}\right)_{\infty}$ by the elastic constants of the surrounding material. The tensor $Q_{t / k l}$ depends on the geome-

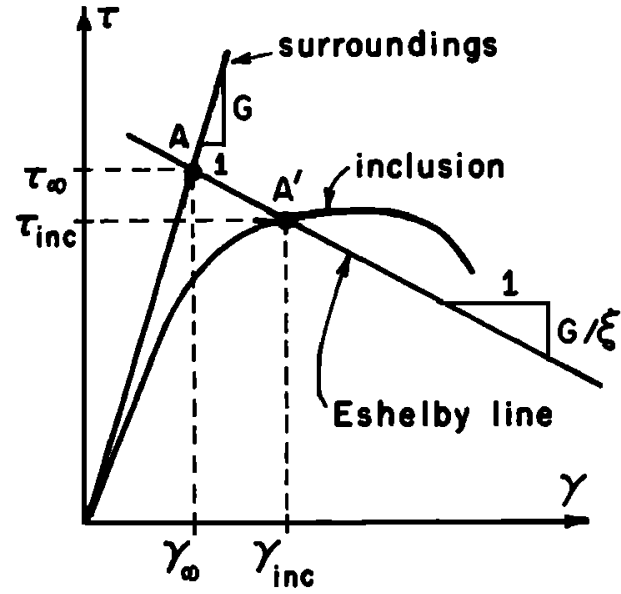

(a)

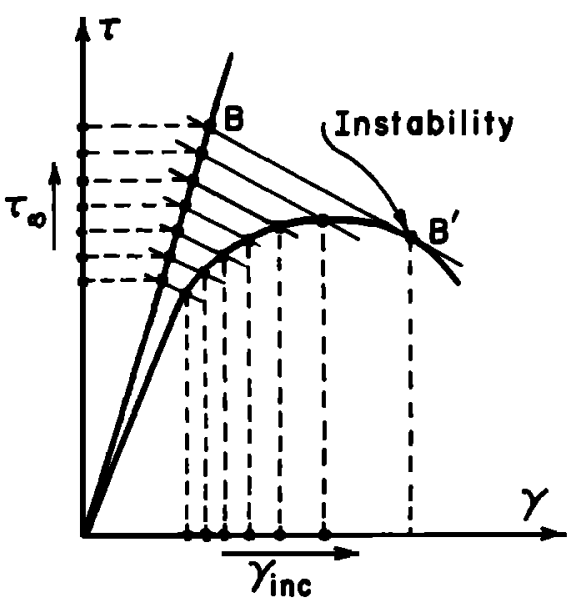

(b)

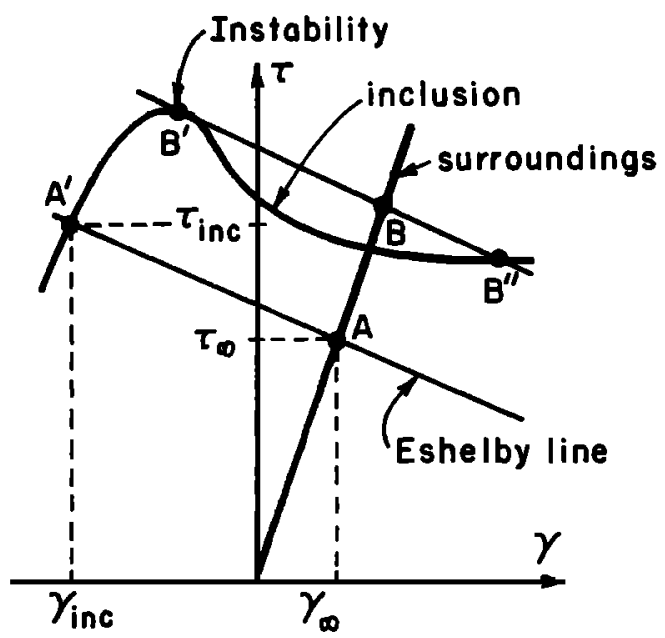

(c)

Fig. 2. Deformation and instability of inhomogeneous zones. (a) Weakened zone. (b) Approach to instability. (c) Sersmic gap zone. 

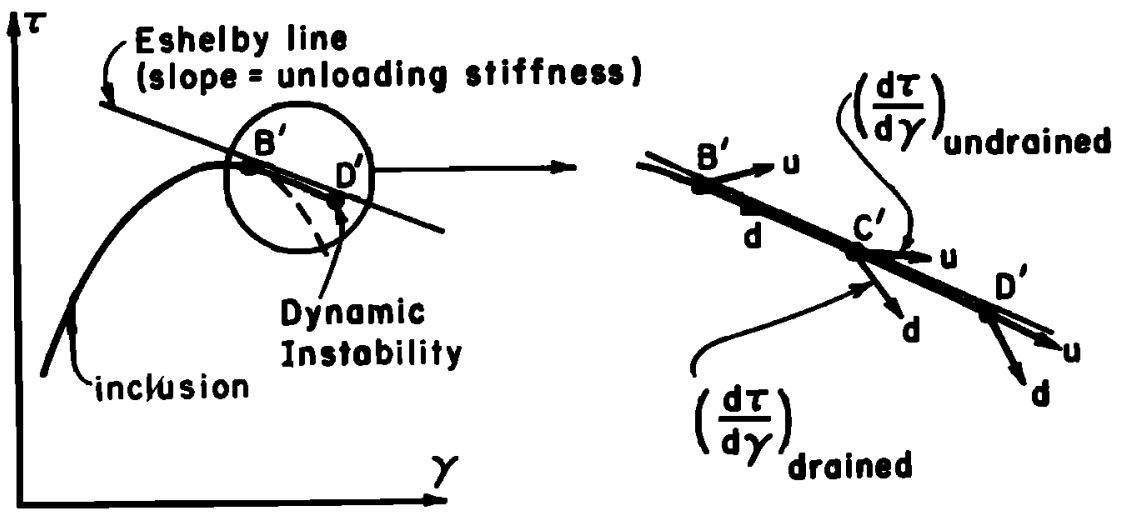

Fig. 3. Stabilization of inhomogeneous zone by dilatant hardening; dynamic instability delayed to point $D^{\prime}$ [Rice, 1977a].

try of the inclusion and the elastic constants of the surrounding material but not on the inclusion properties (as long as they are homogeneous). In particular, (1) pertains whether the inclusion material deforms linearly or nonlinearly. Expressions for $Q_{i j k l}$ in specific cases have been given by $R u d n i c k i$ $[1977 a, b]$.

The state of the inclusion at a particular level of far-field stress may be deduced from a simple graphical construction based on (1). Following Rice et al. [1978], we consider the component of (1) for a single shear stress as illustrated in Figure $1 a$. Then if $\gamma$ denotes the 'engineering' shear strain,

$$
\gamma_{\text {inc }}-\gamma_{\infty}=(\xi / G)\left(\tau_{\infty}-\tau_{\text {inc }}\right)
$$

where $G$ is the shear modulus of the surroundings and $\xi$ a shape factor. This equation is plotted in Figure 2 as the Eshelby line of negative slope $G / \xi$, along with the stress-strain relation for the far field, $\tau_{\infty}=G \gamma_{\infty}$, and the nonlinear $\tau_{\text {inc }}$ versus $\gamma_{1 n c}$ relation for the inclusion. If $\tau_{\infty}$ has its value at point $A$ in Figure 2, the stress in the inclusion is given by the value at point $A^{\prime}$, that is, the intersection of the $\tau_{\text {Inc }}$ versus $\gamma_{\text {inc }}$ curve with Eshelby line through point $A$. As $\tau_{\infty}$ is increased, the successive states in the inclusion traverse the $\tau_{\text {Inc }}$ versus $\gamma_{\text {Inc }}$ curve as illustrated schematically in Figure $2 b$. When $\tau_{\infty}$ has reached its value at point $B$, the Eshelby line is tangent to the inclusion stress strain curve at $\mathbf{B}^{\prime}$. No further increase of $\tau_{\infty}$ can be sustained quasi-statically, and a dynamic runaway of the inclusion shear strain occurs.

The parameter $\xi$ in (2) is given by the following expressions [Rudnicki, 1977a]:

$$
\xi=\frac{2\left(4-5 v_{e}\right)}{7-5 v_{e}}
$$

for a spherical inclusion,

$$
\xi=\frac{4\left(1-\nu_{e}\right)}{\pi\left(2-\nu_{e}\right)} \frac{a}{b}
$$

for a narrow axisymmetrical ellipsoidal inclusion, and

$$
\xi=\frac{\left(1-\nu_{e}\right) a}{\pi b}
$$

for a narrow ellipsoidal inclusion in plane strain, where $\nu_{e}$ is Poisson's ratio in the surrounding material and $a$ and $b$ are the semimajor and semiminor axes of the ellipsoid. (The second expression is a correction by Rice [1977a] of a formula given by Rudnicki [1977a].) An examination of (3)-(5) reveals that the slope of the Eshelby line is less negative for narrow inclusions than it is for nearly spherical ones, and, consequently, runaway occurs nearer to peak stress for the more narrow zones.

Using the construction of Figure $2 b$, we see that an increase of local shear strain rate is predicted as the instability point is approached. In Figure $2 b, \tau_{\infty}$ is increased in equal increments, but it is evident that the corresponding increments of $\gamma_{\text {inc }}$ increase in size. This acceleration of the local strain rate is a general precursory effect, but whether it is sufficient to be observable in terms of surface deformation is unclear.

The graphical construction corresponding to the seismic gap interpretation is shown in Figure $2 c$. Because the inclusion has

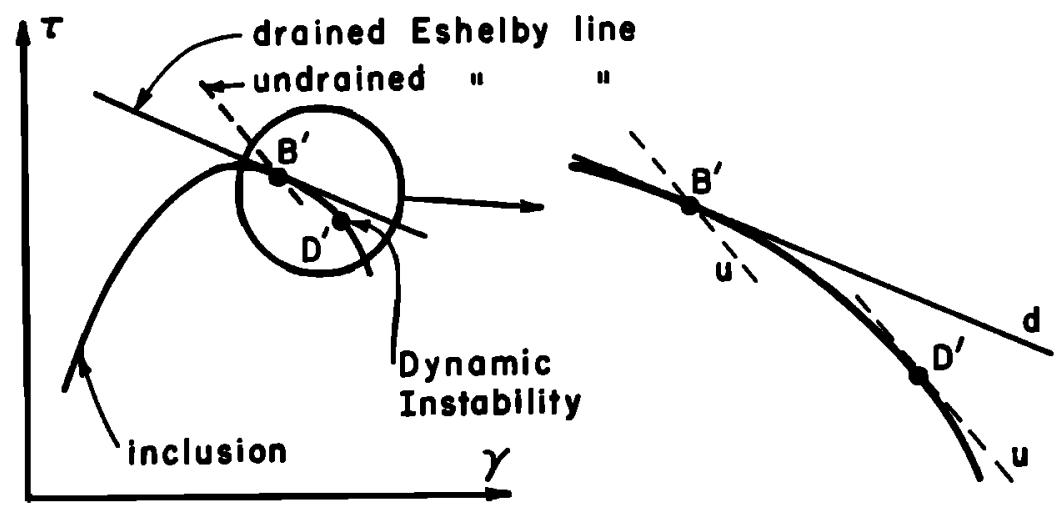

Fig. 4. Stabilization due to time-dependent elastic stiffness. The solid straight line represents the unloading stiffness for drained $(d)$ conditions, and the dashed line that for undrained $(u)$ conditions. Actual stiffiness changes, $d$ to $u$, will be smaller than shown [Rice, 1977a]. 
undergone less strain than the surroundings, the peak of the $\tau_{\text {inc }}$ versus $\gamma_{\text {inc }}$ curve is drawn to the left of the line $\tau_{\infty}=G \gamma_{\infty}$. Therefore the stress sustained by the seismic gap is always greater than $\tau_{\infty}$. That $\gamma_{\text {Inc }}$ is initially negative is inconsequential and results because the strains are measured in relation to those corresponding to the unloaded state in the far field. Otherwise, the analysis is the same as that for the "weakened zone' interpretation of Figures $2 a$ and $2 b$.

The $\tau_{\text {inc }}$ versus $\gamma_{\text {inc }}$ curve in Figure 2 is to be regarded as the relation appropriate to in situ conditions. If the inclusion material dilates in response to shear, as is typical for brittle rock near failure, it must do so against the constraint of the surrounding material. The induced compressive stresses will then inhibit further inelastic deformation and effectively elevate the $\tau_{\text {Inc }}$ versus $\gamma_{1 \mathrm{nc}}$ curve over that for constant mean stress. These effects have been addressed by Rudnicki [1977a] for general deformation states, but it suffices for the purpose of the constructions in Figure 2 simply to regard the $\tau_{\text {inc }}$ versus $\gamma_{\text {inc }}$ curve as drawn so as to incorporate the effects of dilatancy-induced compression. Rudnicki [1977a] has also shown that prior to runaway instability, critical conditions of the type discussed by Rudnicki and Rice [1975] will be met, beyond which the deformation pattern in the inclusion need not remain homogeneous but can begin to bifurcate into localized shear zones. Thus it is appropriate to regard the stress-strain relation of the inclusion as representing the overall response of a nonelastically deforming region even if the deformation is not locally homogeneous.

\section{Effects of Pore Fluid Coupling}

Before examining mathematically the stabilizing effects of coupling of the deformation with pore fluid diffusion, we discuss these effects qualitatively in terms of the schematic illustration of instability in Figure $2 b$. It is evident that Figure $2 b$ may be altered in two ways to prevent instability at B': The local slope of the $\tau_{\text {inc }}$ versus $\gamma_{\text {inc }}$ curve may be increased, or the Eshelby line may be steepened. These correspond to the two mechanisms of stabilization which were discussed in the Introduction, namely, dilatant hardening of the inclusion and stiffening of the elastic response of the surroundings due to undrained conditions.

We consider separately each of the stabilizing mechanisms. First we neglect the time-dependent stiffness of the surroundings and concentrate on dilatant hardening of the inclusion material. The relatively rapid deformation induced near point $B^{\prime}$ will cause the slope $(d \tau / d \gamma)_{\text {inc }}$ to have its elevated, undrained value, which is denoted by $u$ in Figure 3, reproduced from Figure 4 of Rice [1977a]. (We shall later show the relation of this undrained slope to the drained slope and to other constitutive parameters.) Consequently, runaway instability will not occur at $\mathbf{B}^{\prime}$. If $\tau_{\infty}$ deviates only slightly from its value at $B^{\prime}$ (assumed to be the critical value predicted on the basis of drained response), the subsequent deformation will follow the Eshelby line rather than the continuation of the stress-strain curve for drained deformation (shown as a dashed curve in Figure 3). Continued softening, however, will diminish the value of $(d \tau / d \gamma)_{\text {inc }}$ for both drained $(d)$ and undrained $(u)$ responses (e.g., point $C^{\prime}$ in Figure 3). Ultimately, the value of the slope for undrained response falls to the value of that for the Eshelby line, and instability occurs at $D^{\prime}$.

Similarly, we can neglect the changes in the $\tau_{\text {Inc }}$ versus $\gamma_{\text {inc }}$ curve which are induced by dilatant hardening and focus on the stiffness changes due to time-dependent response of the surroundings (Figure 4, reproduced from [Rice, 1977a, Figure
5]). Because of rapid deformation near $B^{\prime}$, the response of the surroundings will not be drained, as indicated by the solid line at $B^{\prime}$ in Figure 4, but rather increments of inclusion and farfield deformation will be related according to the stiffer response appropriate to undrained conditions, as indicated by the dashed line in Figure 4. Although the $\tau_{\text {inc }}$ versus $\gamma_{\text {ine }}$ curve is not tangent to the dashed line at $\mathbf{B}^{\prime}$, continued softening will reduce the value of $(d \tau / d \gamma)_{\text {inc }}$ until it equals the slope of the Eshelby line for undrained response at $D^{\prime}$. Thus instability is delayed beyond $B^{\prime}$, but it occurs at $D^{\prime}$.

In the remainder of the paper we will examine these processes more precisely and concentrate on the time evolution of the system from point $B^{\prime}$ to point $D^{\prime}$ in Figures 3 and 4. For this purpose we require the extension of the Eshelby relations (equation (1)) for application to a fluid-infiltrated solid. This generalization has been accomplished for spherical inclusions by Rice et al. [1978], and in the next subsection we will review their results. Although these relations apply rigorously only for spherical inclusions, the results for a more narrow zone can be approximated by an appropriate modification of parameters.

\section{Eshelby Relations for a Fluid-Infiltrated Elastic Solid}

Rice et al. [1978] observe that when an unbounded fluidinfiltrated elastic solid containing a spherical cavity is subjected to a sudden alteration of pore pressure and surface traction (derivable from a homogeneous stress tensor) at the cavity wall, the wall displaces as if the cavity interior had undergone a homogeneous deformation. This enabled them to generalize the Eshelby relations of (1 and 2) to spherical inclusions in fluid-infiltrated solids, on the assumption that the inclusion is sufficiently permeable by comparison to its surroundings that pore pressure is effectively uniform within it. This assumption seems reasonable in the present context, since the inclusion material is in the dilatant, strain-softening range in the time scale over which we make use of the analysis.

Consequently, the strain and pore pressure fields are homogeneous within a spherical inclusion, and, following Rice et al. [1978], the Eshelby relation of (2) connecting the mismatch of shear stress and strain between the inclusion and the far field is generalized to

$$
\begin{array}{r}
\gamma_{\text {Inc }}(t)-\gamma_{\infty}(t)=\frac{1}{G} \int_{-\infty}^{t}\left\{\xi_{u}+\left(\xi-\xi_{u}\right) f\left[\frac{c\left(t-t^{\prime}\right)}{a^{2}}\right]\right\} \\
\cdot\left[\dot{\tau}_{\infty}\left(t^{\prime}\right)-\dot{\tau}_{\text {Inc }}\left(t^{\prime}\right)\right] d t^{\prime}
\end{array}
$$

where $t$ is time, the overdots denote time differentiation, $G$ is the elastic shear modulus of the material surrounding the inclusion, $a$ is the radius of the inclusion, and $c$ is the diffusivity which appears in the porous medium equations [e.g., Biot, 1941; Rice and Cleary, 1976]. The parameters $\xi$ and $\xi_{u}$ are given by (3) with the value of Poisson's ratio of the surroundings appropriate for drained $(\nu)$ and undrained $\left(\nu_{u}\right)$ deformation, respectively. (Of course, $\xi>\xi_{u}$, since $\nu<\nu_{u}$; some numerical estimates are given in a subsequent table). Consequently, (6) reduces to the forms of (2) which are appropriate for undrained and drained response when the nondimensional function $f\left(c t / a^{2}\right)$ takes on its limiting values for short and long times, respectively:

$$
f(0)=0 \quad f(\infty)=1
$$

The complete variation of $f(\theta)$, where $\theta=c t / a^{2}$, is shown in Figure 5 from Rice et al. [1978, Figure 1] for their $\eta=0.8$ 


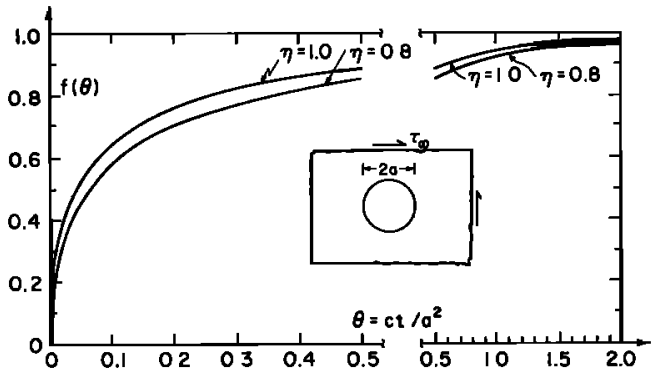

Fig. 5. Function $f(\theta)$ arising in response of spherical cavity to a step shear loading, from Rice et al. [1978].

and 1.0. (The dependence of $f(\theta)$ on $\eta$ is very weak, and $\eta$ itself varies only from 0.8 to 1.0 for representative material parameters.)

It is convenient to have a version of (6) which involves only finite times $t$. Specifically, we assume that prior to $t=0$ there are no excess pore pressures and the inclusion is in equilibrium with its surroundings on the basis of the fully drained elastic properties of the surroundings. That is, prior to $t=0$ the deformations are assumed to take place slowly enough so that (6) reduces to (2) with $\xi$ based on the drained elastic properties, whereas deformation for $t>0$ involves the coupling with diffusion discussed above. In that case it is straightforward to rewrite (6) as

$$
\begin{aligned}
& \gamma_{1 \mathrm{nc}}(t)-\gamma_{\infty}(t)=\frac{\xi}{G}\left[\tau_{\infty}(0)-\tau_{1 \mathrm{nc}}(0)\right] \\
& +\frac{1}{G} \int_{0}^{t}\left\{\xi_{u}+\left(\xi-\xi_{u}\right) f\left[\frac{c\left(t-t^{\prime}\right)}{a^{2}}\right]\right\}\left[\dot{\tau}_{\infty}\left(t^{\prime}\right)-\dot{\tau}_{1 \mathrm{nc}}\left(t^{\prime}\right)\right] d t^{\prime}
\end{aligned}
$$

As mentioned earlier, the response of the inclusion to shear may be coupled to that for compression by dilatancy-induced compressive stresses. Thus for a complete analysis the Eshelby relation for the hydrostatic component of deformation is needed. The appropriate special case of (1) is [Rudnicki, 1977a].

$$
G\left[\epsilon_{\mathrm{inc}}(t)-\epsilon_{\infty}(t)\right]=\stackrel{?}{\mathrm{q}}\left[\sigma_{\mathrm{Inc}}(t)-\sigma_{\mathrm{\infty}}(t)\right]
$$

where the strains $\epsilon$ and stresses $\sigma$ (positive in compression) are the hydrostatic components, $\epsilon=\epsilon_{k k}$, and $\sigma=-\sigma_{k k} / 3$. As shown by Rice et al. [1978], this same relation applies for fluidinfiltrated solids whenever $\sigma_{\infty}$ and $\epsilon_{\infty}$ change with time in a manner consistent with a constant fluid mass content at infinity. This includes the special case which we will consider, for which $\sigma_{\infty}$ and $\epsilon_{\infty}$ are taken to be constant as $\tau_{\infty}$ is increased. It is remarkable that the dilational Eshelby relation of (8) involves only the total hydrostatic stress $\sigma_{\text {inc }}$ within the inclusion and, for a given $\sigma_{\text {inc }}$, is dependent of the pore pressure $p_{\text {inc }}$. This feature follows from the solution to the Biot equations as developed by Rice and Cleary [1976] for simultaneous application of a total stress and pore pressure alteration to the wall of a spherical cavity.

One additional equation is needed to relate the pore fluid pressure $p_{\text {inc }}(t)$ in the inclusion to the fluid mass $m_{\text {lnc }}(t)$ per unit volume within the inclusion. Rice et al. [1978] have obtained this equation by again taking advantage of the solution by Rice and Cleary [1976] for the pore pressure distribution induced outside a spherical cavity by sudden application of a pore pressure to the wall of the cavity. The mass flux into the cavity, as computed from the pore fluid pressure distribution outside the cavity and Darcy's law, is required to balance the rate of fluid mass accumulation at the cavity wall. The result, after extension by superposition to arbitrary time variation, is [Rice et al., 1978]

$$
\begin{aligned}
\dot{m}_{\mathrm{lnc}}(t) & =-\frac{3 \rho k}{a^{2}}\left\{p_{\mathrm{Inc}}(t)-p_{\mathrm{\Phi}}(t)\right. \\
& \left.+\int_{-\infty}^{t} \frac{a}{\left[\pi c\left(t-t^{\prime}\right)\right]^{1 / 2}}\left[\dot{p}_{1 \mathrm{nc}}\left(t^{\prime}\right)-\dot{p}_{\mathrm{\Phi}}\left(t^{\prime}\right)\right] d t^{\prime}\right\}
\end{aligned}
$$

where $\rho$ is the density of the pore fluid in the material surrounding the inclusion ( $\rho$ is assumed to be spatially uniform except for small pressure-dependent changes due to fluid compressibility) and where $k$ is the permeability coefficient of the surroundings, defined so that Darcy's expression for the mass flux rate of pore fluid crossing unit area has the form $\rho k \nabla p$. Sometimes $k$ is written as $k^{\prime}$ (with units of area, measured in darcies) divided by viscosity of the pore fluid [see Rice and Cleary, 1976]. Again, $p_{\infty}$ and $\sigma_{\infty}$ are assumed to vary in a manner that $m_{\infty}$ is constant; for our calculation we will take all to be constant.

Thus we wish to use (7)-(9) to analyze the inclusion model, assuming that $\dot{\sigma}_{\infty}=\dot{p}_{\infty}=0$ and that some uniform tectonic shear stressing rate $\dot{\tau}_{\infty}$ is given. In order to complete the analysis of time-dependent response it is necessary to specify three constitutive relations for the inclusion material which relate its 'strain' quantities $\gamma_{\mathrm{lnc}}, \epsilon_{\mathrm{Inc}}$, and $m_{\mathrm{Inc}}$ to its 'stress' quantities $\tau_{\text {inc }}, \sigma_{\text {inc }}$, and $p_{\text {inc }}$. For simplicity of analysis and clarity of presentation, the effects of dilatant hardening of the inclusion and of the time-dependent stiffness of the surroundings will be considered separately. Constitutive relations for the dilatant hardening analysis are given in the next section, and for that analysis we neglect the time-dependent stiffness of the surroundings implicit in (7), replacing (7) by (2). For the analysis of time-dependent stiffness effects it suffices simply to view $\tau_{\text {inc }}$ as a function of $\gamma_{1 n c}$ without dilatancy effects, as in Figures 2 and 4 , and the analysis can then be based on (7) without explicit consideration of (8) and (9).

Our inclusion model as based on (2) or (7) and (8) and (9) obviously regards the fault zone as being of small extent by comparison to other relevant dimensions. As such, its proximity to the earth's surface is neglected, as is also the nonuniformity of rock properties with depth. These are important limitations (but removable by more elaborate modeling) on the application of our results to large crustal earthquakes.

\section{Stabilization by Dilatant Hardening}

In this section we will examine in detail the manner by which dilatant hardening can delay the onset of rapid failure and give rise to a period of initially slow but accelerating deformation. For convenience the complementary mechanisms due to timedependent stiffness of the surroundings will be neglected. This simplification corresponds to using (2), which we will assume relates rates of stress and strain, rather than (7) as the Eshelby relation for shear. First, however, we will introduce constitutive relations for the inclusion which are intended to model the frictional, dilatant response of brittle rock.

\section{Constitutive Relations}

The constitutive relations employed to describe the response of the inclusion material are analogous to those which were introduced by Rice [1975], generalized to arbitrary deformation states by Rudnicki and Rice [1975], and used by Rudnicki $[1977 a, b]$. These relations are intended to describe both elastic 
response and frictional, dilatant inelastic response of compressed rock due to slip on nominally closed fissure surfaces and to microcrack growth from local tensile stress concentrations.

Consider a material element which is subjected to a hydrostatic stress $\sigma$ (positive in compression), a shear stress $\tau$, and a pore fluid pressure $p$. If the material responds elastically, the shear strain rate $\dot{\gamma}$ and volume strain rate $\dot{\epsilon}$ may be written

$$
\begin{aligned}
& \dot{\gamma}=\dot{\tau} / G \\
& \dot{\boldsymbol{\epsilon}}=-(\dot{\sigma}-\dot{p}) / K-\dot{p} / K_{s}
\end{aligned}
$$

where $G$ is the incremental shear modulus, $K$ is the incremental bulk modulus for drained response, and $K_{g}$ is the bulk modulus of the solid constituents. The combination $\sigma-p(1-K)$ $K_{s}$ ) is the form of the 'effective stress' which has been shown by Nur and Byerlee [1971] to be appropriate for elastic response. In general, however, an increment of deformation involves inelastic response, and these contributions to the strain rate must be added to those of (10) and (11). Following Rice [1977a], we write the complete incremental stress-strain relations as

$$
\begin{aligned}
& \dot{\gamma}=\dot{\tau} / G+[\dot{\tau}-\mu(\dot{\sigma}-\dot{p})] / h \\
& \dot{\epsilon}=-(\dot{\sigma}-\dot{p}) / K-\dot{p} / K_{\mathrm{a}}+\beta[\dot{\tau}-\mu(\dot{\sigma}-\dot{p})] / h
\end{aligned}
$$

where $\mu$ is a friction coefficient and $\beta$ is a dilatancy factor which expresses the ratio of inelastic increments of volume strain to inelastic increments of shear strain. Estimates from experimental results for values of $\mu$ and $\beta$ by Rice [1975], Rudnicki and Rice [1975], and Rudnicki [1977a, b] lie in the ranges $0.5-1.0$ and $0.2-0.5$, respectively. The 'hardening' modulus (or 'softening' if $h<0$ ) is related to the slope of the $\tau$ versus $\gamma$ curve for drained response at constant $\sigma$ by

$$
\left(\frac{\partial \tau}{\partial \gamma}\right)_{\text {dralned }}=\frac{h}{1+h / G} \quad \dot{\sigma}=0
$$

In general, all of the constitutive parameters may vary with the deformation, although the variation in $h$ is typically most substantial.

The form of the effective stress which enters the inelastic contributions in (12) and (13) is $\sigma-p$. Recently, Rice [1977b] has shown rigorously that this is the appropriate form in describing the inelastic response which arises from slip at isolated asperity contacts and/or from local cracking at the tips of sharp microfissures.

One additional constitutive equation is needed for the fluid mass content $m$ per unit volume, which is related to the apparent volume fraction $v$ of pore space by $m=\rho v$, where $\rho$ is the mass density of the homogeneous pore fluid. It is convenient to express $m$ in terms of the stresses by using reciprocity relations [Biot, 1973; Rice, 1975] to deduce the form for the elastic portion of $d v$ and by assuming $d^{p} v=d^{p} \epsilon$ for the inelastic portion [Rice, 1975]. The latter has been shown [Rice, 1977b] to follow rigorously in the same circumstances for which the use of $\sigma-p$ for the inelastic effective stress measure is justified. The result, written in rate form, is

$$
\frac{\dot{m}}{\rho}=\frac{v \dot{p}}{K_{f}}-\left[\frac{1}{K}-\frac{1}{K_{s}}\right](\dot{\sigma}-\dot{p})-\frac{v}{K_{s}} \dot{p}+\beta \frac{[\dot{\tau}-\mu(\dot{\sigma}-\dot{p})]}{h}
$$

where $K_{f}$ is the bulk modulus of the pore fluid $\left(\dot{\rho}=\rho \dot{p} / K_{f}\right)$ and the remaining quantities have been defined previously.
The stiffness of response for shearing under undrained conditions $(\dot{m}=0)$ may now be calculated and compared with the corresponding stiffness for drained conditions (equation (14)). Assuming $\dot{\sigma}=0$ and setting $\dot{m}=0$ in (15) yields

$$
\dot{p}=-\left[\beta K^{\prime} /\left(h+\mu \beta K^{\prime}\right)\right] \dot{\tau}
$$

where

$$
\frac{1}{K^{\prime}}=\frac{1}{K}+\frac{v}{K_{f}}-\frac{1+v}{K_{s}}
$$

By substituting (16) into (13) we obtain the stiffness of response for shear at constant hydrostatic stress under completely undrained conditions:

$$
\left(\frac{d \tau}{d \gamma}\right)_{\text {undrained }}=\frac{h+\mu \beta K^{\prime}}{1+\left(h+\mu \beta K^{\prime}\right) / G} \quad \dot{\sigma}=0
$$

Comparison of (18) with the analogous expression for drained response (equation (14)) reveals that the effective slope of the $\tau$ versus $\gamma$ curve has been augmented by an amount corresponding to the replacement of $h$ by $h+\mu \beta K^{\prime}$. The stiffened response under undrained conditions is depicted schematically in Figure 3 by the arrows labeled $u$. For representative values of $\mu, \beta$, and $K^{\prime}$ this effect is substantial and may be sufficient to cause the slope for undrained response to be positive, while that for drained response is negative.

For heavily fissured rock, $K_{s} \gg K_{,} K_{f} / v$, and therefore

$$
K^{\prime} \simeq K K_{f} /\left(K_{f}+v K\right)
$$

If $K_{f}$ has a value appropriate for liquid water $\left(K_{f}=22 \mathrm{kbar}\right)$ and $v<0.10$, as is typical of brittle rock, $K^{\prime}=K$. High temperatures, low pore pressure, or the presence of entrapped gas may, however, cause $K_{f}$ to be reduced well below $v K$, so that $K^{\prime} \simeq K_{f} / v$ and the dilatant hardening effect disappears in the limit as $K_{,} \rightarrow 0$.

Of course, the foregoing constitutive description is based on the assumption that the state of the pore fluid can be characterized by a single parameter, namely, a pore pressure $p$, which is valid only when deformations are sufficiently slow that there is local pressure equilibrium between all fissure and pore spaces occupying what is regarded as a 'point' in the continuum model of the material. This may not be the case in the presence of rapid deformations; some estimates of equilibrium times and generalizations of the pore pressure concept are considered by Cleary [1977] and $O^{\prime}$ Connell and Budiansky [1977]. Indeed, some discussions of dilatancy [e.g., Nur, 1972] consider the possibility that the effect may be so strong as to open substantial vapor-filled, or 'dry,' crack space in rock that is otherwise liquid saturated. Such a concept seems to be widely associated with the term 'dilatancy' and is consistent with alterations of seismic wave speeds. But it is important to realize that far milder dilatancy, insufficient to cause the opening of dry crack space and thus to affect seismic wave transmission, may nevertheless be present during a failure process and, possibly, be a major factor in controlling the time scale of that failure through the processes to be described.

\section{Dilatant Hardening and Instability}

In order to apply the results of the last section to the inclusion problem, we adopt (12), (13), and (15) as the constitutive laws for the inclusion material and employ them in conjunction with the Eshelby relations (2), (8), and (9). The analysis follows that of Rudnicki [1977b, chapter 2]. Thus the 
relations between rates of stress and strain are, from (2) and (8),

$$
\begin{aligned}
\dot{\gamma}_{\mathrm{Inc}}(t)-\dot{\gamma}_{\Phi}(t) & =\frac{\xi}{G}\left[\dot{\tau}_{\Phi}(t)-\dot{\tau}_{\mathrm{inc}}(t)\right] \\
\dot{\epsilon}_{\mathrm{Inc}}(t) & =(3 / 4 G) \dot{\sigma}_{\mathrm{Inc}}(t)
\end{aligned}
$$

where we have assumed in (21) that $\dot{\sigma}_{\infty}=0$. If (12) and (13) are combined with (20) and (21) to eliminate $\dot{\tau}_{\text {1nc }}$ and $\dot{\sigma}_{\text {inc }}$, the results are

$$
\begin{aligned}
\dot{\gamma}_{\mathrm{inc}}=\dot{\gamma}_{\infty}\left(1+\frac{\kappa G}{h-h_{d}}\right)+\frac{\mu \dot{p}_{\mathrm{nc}} \kappa \alpha\left(1+4 G / 3 K_{s}\right)}{h-h_{d}} \\
\dot{\epsilon}_{1 \mathrm{nc}}=\frac{\beta G \alpha}{h-h_{d}} \dot{\gamma}_{\infty}+\frac{\alpha \dot{p}_{\mathrm{lnc}}}{K} \\
\cdot\left[1+\frac{K}{K_{s}}-\frac{\mu \beta K \alpha\left(1+4 G / 3 K_{s}\right)}{h-h_{d}}\right]
\end{aligned}
$$

where $\kappa=\xi /(1+\xi)$ and $\alpha=1 /(1+4 G / 3 K)=(1+\nu) / 3(1-$ $\nu)$ and we have assumed for simplicity that the elastic moduli of the inclusion are identical with those of the surroundings. (Rudnicki [1977b] has given the corresponding expressions if there is elastic mismatch between the inclusion and the surroundings but assuming effectively incompressible solid constituents for the inclusion $\left(K_{s} \rightarrow \infty\right.$.) The parameter $h_{d}$ is the value of $h$ for which the ratio $\dot{\gamma}_{1 n c} / \dot{\gamma}_{\infty}$ in (22) becomes unbounded under completely drained conditions $(\dot{p}=0)$ and corresponds to the onset of dynamic runaway (point $B^{\prime}$ in Figure 3):

$$
h_{t}=-G /(1+\xi)-\mu \beta K /(1+3 K / 4 G)
$$

The first term on the right in (24) would result from equating the right-hand side of (14) to the slope of the Eshelby line $(-G / \xi)$. whereas the second term reflects the inhibiting effect of dilatancy-induced compressive stresses. In other words, the hardening modulus corresponding to the in situ stress-strain curve of the inclusion is

$$
H=h+\mu \beta K /(1+3 K / 4 G)
$$

In order to demonstrate that the stiffened response to undrained deformation (compare (18) with (14) or $u$ with $d$ in Figure 3) can in fact stabilize against runaway at $h=h_{d}$, we again consider completely undrained conditions. First, using (13) we can rewrite (15) as

$$
\frac{\dot{m}}{\rho}=\frac{v \dot{p}}{K_{f}}+\frac{\left(\dot{\sigma}_{1 \mathrm{nc}}-v \dot{p}\right)}{K_{\mathrm{s}}}+\dot{\epsilon}_{\text {inc }}
$$

where we here and subsequently drop the designation 'inc' from $\dot{m}$ and $\dot{p}$. Eliminating $\dot{\epsilon}_{1 \mathrm{nc}}$ and $\dot{\sigma}_{1 \mathrm{nc}}$ from (25) by means of (21) and (23) yields the rate of pore fluid pressure decrease in the inclusion

$$
(\dot{p})_{\text {undrained }}=\frac{-\beta G K_{f}^{\prime} \dot{\gamma}_{\infty}}{h-h_{d}+\alpha \mu \beta K_{f}^{\prime}\left(1+4 G / 3 K_{s}\right)}
$$

where

$$
\frac{1}{K_{f}^{\prime}}=\frac{v}{\alpha K_{f}} \frac{\left(1-K_{f} / K_{s}\right)}{\left(1+4 G / 3 K_{s}\right)}+\frac{1}{K}\left(1-\frac{K}{K_{s}}\right)
$$

Substituting (26) into (22) yields

$$
\dot{\gamma}_{1 \mathrm{nc}}=\dot{\gamma}_{\infty}\left[1+\frac{\kappa G}{h-h_{d}+\alpha \mu \beta K_{f}^{\prime}\left(1+4 G / 3 K_{8}\right)}\right]
$$

and demonstrates that the ratio $\dot{\gamma}_{\text {inc }} / \dot{\gamma}_{\infty}$ remains finite at $h=$ $h_{d}$. Even if the rock mass is constrained to deform in a completely undrained fashion, however, $\dot{\gamma}_{\text {inc }} / \dot{\gamma}_{\infty}$ does become unbounded at

$$
h=h_{u} \equiv h_{d}-\alpha \mu \beta K_{f}^{\prime}\left(1+4 G / 3 K_{s}\right)
$$

which corresponds to point $D^{\prime}$ in Figure 3. The discussion of (28) in cases of limiting behavior for $K_{f}^{\prime}$ follows the earlier remarks for $K^{\prime}$.

Although dilatant hardening can stabilize against the onset of rapid failure at $h=h_{d}$, the subsequent deformation is selfdriving [Rudnicki, 1977b]. More precisely, Rudnicki [1977b] showed that in a segment of constant $h$ (and other constitutive parameters), any perturbation of $\gamma_{\text {inc }}$ from its equilibrium value corresponding to some fixed $\tau_{\mathrm{s}}$ is stable if $h>h_{d}$ (i.e., prior to point $B^{\prime}$ in Figure 3), in the sense that the perturbation decays with increasing time. However, when $h_{d}>h>h_{u}$ (i.e., for states between points $B^{\prime}$ and $D^{\prime}$ in Figure 3), perturbations in $\gamma_{1 n c}$ grow exponentially with increasing time, and the rate of growth becomes unbounded when $h=h_{u}$. We define the precursor time as the time which elapses from the onset of this period of self-driven deformation at $B^{\prime}$ to dynamic instability at $\mathrm{D}^{\prime}$.

In order to determine the evolution of the inclusion state and thus the precursor time it is necessary to solve the system of equations consisting of the constitutive laws (12), (13), and (15) and the Eshelby relations (20), (21), and (9). The number of governing equations may, however, be reduced to two: (22) and a single equation for the alteration in pore fluid pressure $p(t)$. The latter can be obtained by using (21) and (23) to eliminate $\dot{\sigma}_{\text {Inc }}$ and $\dot{\epsilon}_{\text {inc }}$ from (25), which then becomes

$$
\frac{\dot{m}}{\rho}=\frac{\alpha\left(1+4 G / 3 K_{s}\right)}{K_{f}^{\prime}\left(h-h_{d}\right)}\left[\beta G \dot{\gamma}_{\infty}+\dot{p}\left(h-h_{u}\right)\right]
$$

where $K_{f}^{\prime}$ and $h_{u}$ have been defined in (26) and (28). Combining (9) with (29) yields

$$
\begin{array}{r}
t_{D} \dot{p}(t)=-\frac{\beta K_{f}^{\prime} G \dot{\gamma}_{\infty} t_{D}}{h-h_{u}}-\left[\frac{3 K_{f}^{\prime}}{\alpha\left(1+4 G / 3 K_{a}\right) N}\right] \frac{h-h_{d}}{h-h_{u}} \\
\times\left\{p(t)+\int_{0}^{t} \frac{a}{\left[\pi c\left(t-t^{\prime}\right)\right]^{1 / 2}} \dot{p}\left(t^{\prime}\right) d t^{\prime}\right\}
\end{array}
$$

where $t_{D}=a^{2} / c$ is the diffusion time, $p(t)$ is now measured in relation to the ambient value which exists in response to the constant hydrostatic stress at infinity, and the lower limit of the integral has been chosen by taking $p(t)=0$ for $-\infty<t \leq$ 0 . The parameter $N=c / k$ is an additional elastic modulus which Rice and Cleary [1976] have shown may be written as

$$
N=\frac{2 G(1-\nu) B^{2}\left(1+\nu_{u}\right)^{2}}{9\left(1-\nu_{u}\right)\left(\nu_{u}-\nu\right)}
$$

where $\nu_{u}$ is Poisson's ratio for undrained response and $B$ is the ratio of pore fluid pressure decrease to mean normal stress increase for an increment of undrained deformation.

The dependence of $h$ and, in general, of all of the constitutive parameters on the deformation couples (30) to (22). In solving these equations we will assume, however, that the variation in all the parameters except $h$ is small enough that they may be treated as constant. Although this may be a poor approximation in the case of $\beta$ and $K_{f}$, so little is known about the details of their variation that assuming they are constant seems justified in the interests of simplicity. The simple numerical procedure which was used to solve (30) and (22) is out- 


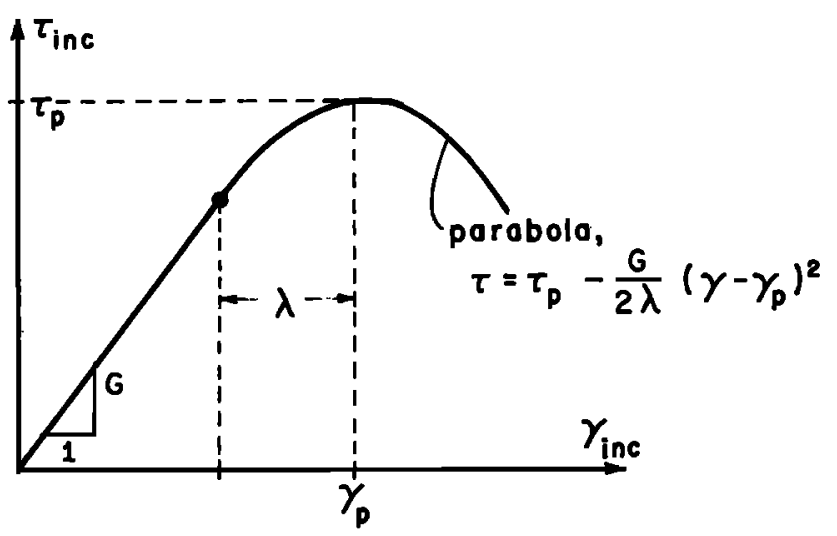

Fig. 6. Inclusion stress-strain relation.

lined in Appendix 1. Results of the calculations and a discussion of the constitutive parameters which were used are presented in the following subsection.

\section{Numerical Results for \\ Precursor Time}

As mentioned earlier, the precursor time is defined as the time which elapses from the onset of self-driven deformation at point $B^{\prime}$ (Figure 3 ) to instability at $D^{\prime}$. For the calculation we assume that the in situ $\tau_{1 \mathrm{nc}}$ versus $\gamma_{\text {inc }}$ curve has the form of a linear segment of slope $G$ up to an elastic limit and that this segment is connected smoothly to the following parabola (Figure 6):

$$
\tau_{\text {inc }}=\tau_{p}-\frac{G}{2 \lambda}\left(\gamma_{\text {inc }}-\gamma_{p}\right)^{2}
$$

where $\gamma_{p}$ is the strain at peak stress, $\lambda$ is the difference between $\gamma_{p}$ and the strain at the elastic limit, and $G$ is the slope at the elastic limit. Because this represents the in situ stress-strain curve, the value of $H=h+\mu \beta K /(1+3 K / 4 G$ ) (see following (24)) is related to $d \tau_{\text {inc }} / d \gamma_{\text {inc }}$ by

$$
\frac{d \tau_{\mathrm{inc}}}{d \gamma_{\mathrm{inc}}}=\frac{H}{1+H / G}
$$

In particular, we choose $\tau_{p}=1 \mathrm{kbar}, \gamma_{p}=6.25 \times 10^{-3}, \lambda=$ $2.5 \times 10^{-3}$, and $G=200 \mathrm{kbar}$. For the remaining material parameters we assume $\nu=0.2, \mu=0.6, \beta=0.3, N=2.0 G, K_{r}^{\prime}$

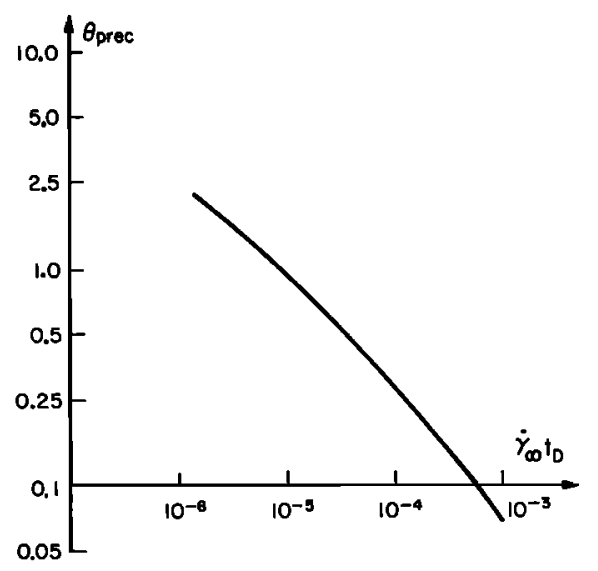

Fig. 7. Predicted precursor time $t_{\mathrm{prec}} / t_{D}$ as a function of the nondimensional far-field strain rate $\dot{\gamma}_{\boldsymbol{c}} t_{D}$. For values of material parameters, see the text.

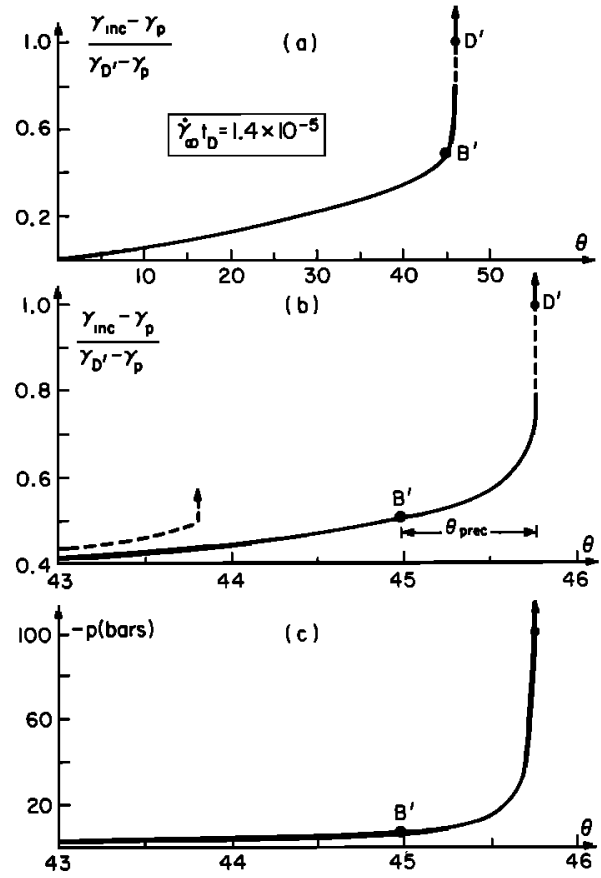

Fig. 8. $(a, b)$ History of postpeak straining of the inclusion and $(c)$ the decrease of pore fluid pressure in the inclusion. Computed for $\dot{\gamma}_{\odot} t_{D}$ $=1.4 \times 10^{-5}$. Points $\mathrm{B}^{\prime}$ and $\mathrm{D}^{\prime}$ are as in Figure 3 , and $\gamma_{D^{\prime}}$ is the value of the strain at point $\mathrm{D}^{\prime}$.

$=1.45 G$, and $K_{8}=5 G$. The value of $N$ corresponds to using $\nu_{u}$ $=0.37$ and $B=0.8$ and is in the range, though toward the lower end, of values inferred from Rice and Cleary [1976, Table 1]. The value of $K_{f}^{\prime}$ is based on an initial porosity of $v=$ 0.01 assuming $K_{f}=22 \mathrm{kbar}$. The calculation was begun at peak stress, where it was assumed the alteration in pore fluid
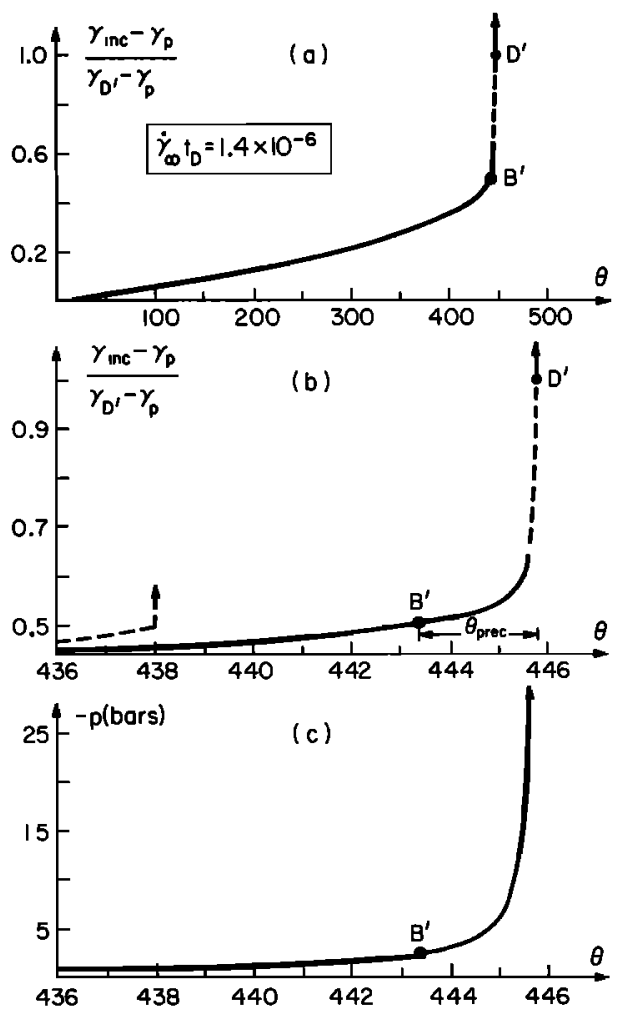

Fig. 9. Same as Figure 8 except $\dot{\gamma}_{\sigma^{\circ}}=1.4 \times 10^{-8}$. 
TABLE 1. Predicted Precursor Times Based on Dilatant Hardening of a Spherical Zone

\begin{tabular}{|c|c|c|}
\hline$a=1 \mathrm{~km}$ & $a=3 \mathrm{~km}$ & $a=5 \mathrm{~km}$ \\
\hline $\begin{aligned} t_{D} & =11.6 \\
t_{\mathrm{prec}} & =55.4\end{aligned}$ & $\begin{aligned} c & =1 \mathrm{~m}^{2} / \mathrm{s} \\
t_{D} & =104 \\
t_{\mathrm{prec}} & =234\end{aligned}$ & $\begin{aligned} t_{D} & =289 \\
t_{\mathrm{prec}} & =411\end{aligned}$ \\
\hline $\begin{aligned} t_{D} & =116 \\
t_{\text {prec }} & =238\end{aligned}$ & $\begin{aligned} c & =0.1 \mathrm{~m}^{2} / \mathrm{s} \\
t_{D} & =1042 \\
t_{\mathrm{prec}} & =844\end{aligned}$ & $\begin{aligned} t_{D} & =2894 \\
t_{\mathrm{prec}} & =1418\end{aligned}$ \\
\hline & Scholz et al. [1973] & \\
\hline$t_{\mathrm{prec}} \approx 8$ & & $t_{\mathrm{prec}} \approx 200$ \\
\hline
\end{tabular}

Values of $t_{\text {prec }}$ and $t_{D}$ are in days; $t_{\text {prec }}$ is the time for the transition from $B^{\prime}$ to $D^{\prime}$ in Figure 3. The precursor time for $a=1, c=1 \mathrm{~m}^{2} / \mathrm{s}$ was estimated by extrapolation of the curve in Figure 7. Data used were the following: tectonic stress rate $\dot{\tau}_{\mathrm{c}}=1 \mathrm{bar} / \mathrm{yr}$; peak strain parameter (Figure 6) $\lambda=0.0025$; frictional coefficient $\mu=0.6$; dilatancy factor $\beta=0.3$; initial porosity $v=0.01 ; N=c / k=2.0 G ; K_{\mathrm{a}}=5.0 G$; $K_{f}=22 \mathrm{kbar} ; G=200 \mathrm{kbar} ; v=0.2$.

pressure (taken to be zero at the elastic limit) was given by the steady state term in (30):

$$
p(H=0)=-\frac{\beta \alpha\left(1+4 G / 3 K_{s}\right) N}{3\left(-H_{d}\right)} \dot{\tau}_{\infty} t_{D}
$$

where $H_{d}=-G /(1+\xi)$.

The dimensionless precursor time $\theta_{\text {prec }}=t_{\text {prec }} / t_{D}$ is shown in Figure 7 as a function of the dimensionless far-field strain rate $\dot{\gamma}_{\infty} t_{D}$. It is evident that the predicted precursor time does not simply scale with $\dot{\gamma}_{\infty} t_{D}$. In particular, although $t_{\text {prec }}$ increases with $t_{D}=a^{2} / c$, the increase is not proportional.

The time history of postpeak straining is shown in Figures 8 and 9 for $\dot{\gamma}_{\infty} t_{D}=1.4 \times 10^{-5}$ and $1.4 \times 10^{-6}$, respectively. The strain is given in terms of the parameter $\left(\gamma_{\text {inc }}-\gamma_{p}\right) /\left(\gamma_{D^{\prime}}-\gamma_{p}\right)$, where $\gamma_{p}$ is the strain at peak stress and $\gamma_{D^{\prime}}$ is the strain at point $\mathrm{D}^{\prime}$ in Figure 3. Point $\mathrm{B}^{\prime}$ corresponds to the onset of the period of self-driven deformation. The dashed portion of the solid curve indicates where the numerical caluclation was truncated (see Appendix 1). The fully dashed curves in Figures $8 b$ and $9 b$ indicate for comparison the strain history in the $a b-$ sence of pore fluid effects. Those were calculated by setting $\dot{p}_{\text {inc }}$ $=0$ in (22) and integrating for $\gamma_{i n c}$ as a function of time. It is evident that dilatant hardening not only delays the onset of instability but also gives rise to a more dramatic acceleration of precursory strain. In Figures $8 c$ and $9 c$ the decrease in the pore fluid pressure of the inclusion is shown. It is noteworthy that the decrease is extremely rapid but occurs very close to final instability. This suggests that precursory phenomena which may be associated with the rapid decrease in pore fluid pressure may occur over a time period which is substantially less than the period of self-driving deformation.

The results are shown in dimensional form in Table 1 . We consider three values for the radius of the sphere; namely, $a=$ $\mathrm{I}, 3$, and $5 \mathrm{~km}$; and two values of the diffusivity, $c=1$ and 0.1 $\mathrm{m}^{2} / \mathrm{s}$. The larger value of the diffusivity was suggested by $D . L$. Anderson and J. H. Whitcomb [1975] as being reasonable for shallow earthquake zones, whereas $c=0.1 \mathrm{~m}^{2} / \mathrm{s}$ is more in accord with well head measurements near the San Andreas Fault [Kovach et al., 1975]. The value of $\dot{\gamma}_{\infty}$ was chosen to correspond to $\dot{\tau}_{\infty}=1 \mathrm{bar} / \mathrm{yr}$, i.e., $\dot{\gamma}_{\infty}=\dot{\tau}_{\infty} / G$, which is consistent with the centennial occurrence of a large earthquake having a stress drop of 100 bars. Strain accumulation measurements along the San Andreas Fault near Palmdale [Prescont and Savage, 1976] suggest a value of $\dot{\tau}_{\infty}$, which is roughly an order of magnitude smaller, so that values of $\dot{\gamma}_{\infty} t_{D}$ which are smaller than those plotted in Figure 7 may be relevant. However, because of the expense of computation for small values of $\dot{\gamma}_{\mathrm{o}} t_{D}$, the precursor time for $a=1 \mathrm{~km}$ and $c=1 \mathrm{~m}^{2} / \mathrm{s}$ was simply estimated by extrapolating the curve in Figure 7. The values in Table 1 again indicate that although $t_{\text {prec }}$ increases with the diffusion time $a^{2} / c$, the increase is not proportional. A tenfold increase in $a^{2} / c$ corresponds to an increase of roughly 4-5 times in $t_{\text {prec }}$.

The last row of Table 1 gives the 'best fit' correlation by Scholz et al. [1973] of precursory times (based on $V_{p} / V_{s}$ anomalies, radon emission, and crustal movements) with the length of aftershock zone. Individual data points may differ by a factor of approximately 2 from this time. The values in Table 1 identify $2 a$ with the length of the aftershock zone. Comparison of the calculated with the observed precursor times indicates that the calculated values are generally larger than but perhaps not inconsistent with those observed. The observation of seismic anomalies, for example, may be possible only toward the later stages of what is referred to here as the precursory period. This interpretation is consistent with the earlier remark concerning the prediction that rapid decrease of pore fluid pressure occurs relatively near final instability.

The values of the material parameters which were used in the calculations were chosen to be consistent with the existing laboratory and field data. In order to assess the magnitude of the effect that variations in the more uncertain of these parameters might have, we have performed a few additional calculations for alternative values of the dilatancy factor $\beta$, the peak strain parameter $\lambda$, and the fluid bulk modulus $K_{f}$. The calculations were carried out for $\dot{\gamma}_{c t_{D}}=1.4 \times 10^{-5}$, which corresponds to $\dot{\tau}_{\infty}=1 \mathrm{bar} / \mathrm{yr}, a=3 \mathrm{~km}$, and $c=0.1 \mathrm{~m}^{2} / \mathrm{s}$. The results are summarized in Table 2 .

The values of $\beta$ which were quoted earlier were estimated from laboratory data for tests on intact rock. Because laboratory tests have indicated that dilatancy may diminish somewhat with cyclic loading [e.g., Scholz and Kranz, 1974; Zoback and Byerlee, 1975] and with increased confining stress [Brace et al., 1966], smaller values of $\beta$ may be more representative of in situ conditions. Table 2 shows the calculated dimensionless precursor time for values of $\beta$ equal to one half and one quarter of the value used for other computations $(\beta=0.3)$. Reduction of $\beta$ by half apparently reduces $t_{\text {prec }} / t_{D}$ by slightly

TABLE 2. Eflect of Variations in the Dilatancy Factor $\beta$, the Peak Strain Parameter $\lambda$, and the Fluid Bulk Modulus $K_{f}$ on $\theta_{\text {prec }}$

\begin{tabular}{ll}
$\begin{array}{c}\text { Material } \\
\text { Parameter Value }\end{array}$ & $\theta_{\text {prec }}$ \\
\hline Parameter $\beta$ & \\
0.075 & 0.16 \\
0.15 & 0.39 \\
0.30 & 0.81 \\
& \\
Parameter $\lambda$ & 0.56 \\
0.00125 & 0.81 \\
0.0025 & 1.13 \\
0.0050 & \\
& \\
Parameter $K_{f}$, kbar & 0.44 \\
2.2 & 0.76 \\
11 & 0.81 \\
22 &
\end{tabular}

All calculations were for $\dot{\gamma}_{\omega} t_{D}=1.4 \times 10^{-5}$ and values of other parameters as in Table 1. 
more than half. Note, however, that even for $\beta=0.075, \theta_{\text {prec }}=$ 0.16 corresponds to approximately 170 days in real time for $a$ $=3 \mathrm{~km}$ and $c=0.1 \mathrm{~m}^{2} / \mathrm{s}$. This observation emphasizes that even a very small amount of dilatancy may have quite substantial effects of the kind described here.

The value of the peak strain parameter $\lambda$ is one of the most uncertain in the analysis, since laboratory investigations of postpeak behavior have been relatively sparse. Fortunately, the calculation does not appear to be especially sensitive to the value of $\lambda$. The entries in Table 2 suggest that decreasing $\lambda$ by half reduces $\theta_{\text {prec }}$ by about $30 \%$.

In the calculations for Table $I$ and Figure 7 the bulk modulus of the fluid $K_{r}$ was assumed to have a value appropriate for liquid water, i.e., $K_{f} \simeq 22$ kbar. We remarked earlier, however, that high temperatures, low pressures, or the presence of dissolved gases may reduce $K_{f}$. Table 2 shows the calculated precursor times for $K_{t}=11$ and $2.2 \mathrm{kbar}$. These correspond to values of $K_{f}^{\prime} / G\left(K_{f}^{\prime}\right.$ is defined following (26)) of 1.2 and 0.5 by comparison with $K_{f}^{\prime} / G=1.45$ for $K_{f}=22 \mathrm{kbar}$. We remark that an equivalent reduction of $K_{f}^{\prime}$ to $0.5 G$ can be accomplished by an increase of porosity from $v=0.01$ to $v=0.1$. The reduction of $K_{f}$ by half reduces $\theta_{\text {prec }}$ by only about $6 \%$, and for $K_{f}=2.2 \mathrm{kbar}$ the predicted precursor time is decreased by slightly less than half.

In all of the calculations it has been assumed that the hardening modulus was the only material parameter which varied substantially with the deformation. A more detailed calculation should consider the variation of other material parameters. In particular, appreciable alteration of the dilatancy factor $\beta$ or of the bulk modulus of the pore fluid $K_{f}$ will probably be important. It seems evident that there must exist a limit to the amount of dilatancy which a rock may undergo. Thus it is likely that $\beta$ may decrease at large strains and that the stabilizing effect of dilatant hardening may be limited by the attainment of a least dense state corresponding to no further dilatancy. This is consistent with the measurements by Crouch [1970], which indicated a decrease in the rate of dilatancy relative to the axial strain after some amount of deformation past peak stress, although dilatancy did continue throughout the postpeak regime.

A second limiting effect, which was mentioned earlier, is the increase in fluid compressibility. For the calculations performed here, the decrease in the pore fluid pressure from the ambient level which was assumed to exist when the inclusion material was at the elastic limit did not approach 100 bars until very near instability. The variations shown in Figures $8 c$ and $9 c$ are typical, though the decreases became larger more rapidly for larger values of $\dot{\gamma}_{\infty} l_{D}$. Because the lithostatic pressure at $5-\mathrm{km}$ depth is about 500 bars, the calculations suggest that substantial decreases of $K_{f}$ may be difficult to achieve by pore pressure decreases alone, at least until very near instability. It should be emphasized that 'very near instability' in terms of the dimensionless precursor time may be of the order of days or even weeks in real time, as can be seen from Table 1. Furthermore, elevated temperatures and exsolution of trapped gas may contribute significantly to reducing $K_{r}$.

The calculations also apply rigorously only for spherical inclusions. A preliminary analysis by Rudnicki [1977b] for á flat elliptical inclusion in plane strain indicates that the time scale of stabilizing effects due to dilatant hardening is very much shorter for narrow zones. In particular, Rudnicki [1977b] derived an equation whose asymptotic long-time characteristics were similar to those of (30), except that the appropriate length which entered the diffusion time $L^{2} / c$ was the short axis of the ellipse. This suggests that for an axisymmetric ellipsoid of aspect ratio $a / b=20$, the diffusion time is $1 / 400$ of that for a sphere of radius $a$. A more complete analysis is needed to determine the extent to which dilatant hardening effects are influenced by geometry.

\section{Stabilization by Time-Dependent Stiffness OF SURROUNDINGS}

For the analysis of this mechanism we neglect any suctions induced in the pore fluid within the inclusion and assume that the inclusion response to shearing can be represented by a plot of $\tau_{1 \mathrm{nc}}$ versus $\gamma_{1 \mathrm{nc}}$ as in Figures 2 and 4. As remarked earlier, this plot may be thought of as representing in situ conditions and hence as including effects of an increasing mean stress $\sigma$ induced by the constraint of the surroundings against inelastic dilation of the inclusion material. Thus letting

$$
\tau_{\text {lnc }}=F\left[\gamma_{\text {Inc }}\right]
$$

describe the stress-strain relation of the inclusion material, the mathematical problem is to solve (7) subject to this relation for a given history $\tau_{\infty}(t)$. Since we assume in deriving $(7)$ that there is equilibrium under fully drained conditions for $t \leq 0$, the state at $t=0$ must satisfy (2), namely,

$$
\gamma_{\text {inc }}(0)-\gamma_{\infty}(0)=\frac{\xi}{G}\left[\tau_{\infty}(0)-\tau_{\text {inc }}(0)\right]
$$

and this may be used to simplify the right side of (7). Hence by using (32) and $\tau_{\infty}=G \gamma_{\infty},(7)$ becomes the nonlinear integral equation

$$
\begin{aligned}
\gamma_{\mathrm{Inc}}(t)= & \gamma_{\mathrm{Inc}}(0)+\frac{1}{G}\left[\tau_{\infty}(t)-\tau_{\infty}(0)\right] \\
& +\frac{1}{G} \int_{0}^{t}\left\{\xi_{u}+\left(\xi-\xi_{u}\right) f\left[\frac{c\left(t-t^{\prime}\right)}{a^{2}}\right]\right\} \\
& \cdot\left\{\dot{\tau}_{\infty}(t)-F^{y}\left[\gamma_{\text {inc }}\left(t^{\prime}\right)\right] \dot{\gamma}_{\mathrm{lnc}}\left(t^{\prime}\right)\right\} d t^{\prime}
\end{aligned}
$$

where $F^{\prime}\left[\gamma_{1 \mathrm{nc}}\right]=d F\left[\gamma_{1 \mathrm{nc}}\right] / d \gamma_{\text {Inc }}$. This equation applies for $t \geq$ $0 ; \tau_{\infty}(t)$ is regarded as given, and $\gamma_{\text {inc }}(t)$ is to be determined.

This equation is rigorous for a spherical inclusion with $\xi$ and $\xi_{u}$ calculated from (3) using the drained and undrained Poisson ratios, $\nu$ and $\nu_{u}$, respectively, for $\nu_{e}$. As an approximation, however, we shall also assume that (33) applies to other types of inhomogeneous zones, specifically to the flattened axisymmetric ellipsoidal zone of semimajor axis $a$, for which $\xi$ and $\xi_{u}$ are calculated from (4). For the function $F\left[\gamma_{\mathrm{inc}}\right]$ of (32) we use the parabolic stress-strain relation in Figure 6. We assume that the inclusion strain is at the peak of the curve when $t=0, \gamma_{\mathrm{inc}}(0)=\gamma_{p}$ and that the remote tectonic stress is increased at a constant rate $\dot{\tau}_{\mathrm{o}}$ for all subsequent time.

Equation (33) must be solved numerically, and the details are explained in Appendix 2. Essentially, we find that the solution can be put in the dimensionless form

$$
\left[\gamma_{\text {inc }}(t)-\gamma_{p}\right] /\left[\gamma_{D^{\prime}}-\gamma_{p}\right]=g\left(\theta ; R, \xi / \xi_{u}, \xi\right)
$$

Here $\gamma_{D^{\prime}}$ is the inclusion strain at the point corresponding to $D^{\prime}$ in Figure 4 (i.e., the undrained instability point, at which dynamic instability occurs), $\theta=c t / a^{2}=t / t_{D}$ as before, and $R$ is dimensionless measure of the tectonic stressing rate, namely,

$$
R=\xi(1+\xi)\left(a^{2} / c\right)\left(\dot{\tau}_{\infty} / G \lambda\right)
$$

Apart from the factor $\xi(1+\xi)$, which depends on the shape of the inclusion, $R$ can be interpreted as the ratio of the charac- 
TABLE 3. Estimates of Drained and Undrained Poisson Ratios for Fluid-Infiltrated Solids With Cracklike Pore Spaces

\begin{tabular}{ccccccc}
\hline & \multicolumn{2}{c}{ Poisson Ratio } & & \multicolumn{2}{c}{ (Undrained Stiffness)/(Drained Stiffness) $=\xi / \xi_{u}$} \\
\cline { 2 - 3 } $\begin{array}{c}\text { Crack Density } \\
\text { Parameter } N r^{3}\end{array}$ & $\begin{array}{c}\text { Drained } \\
\nu\end{array}$ & $\begin{array}{c}\text { Undrained } \\
\nu_{u}\end{array}$ & & $\begin{array}{c}\text { Narrow } \\
\text { Elliptical } \\
\text { Cylinder }\end{array}$ & $\begin{array}{c}\text { Narrow } \\
\text { Axisymmetrical } \\
\text { Ellipsoid }\end{array}$ & Sphere \\
\hline 0 & 0.25 & 0.25 & & 1.00 & 1.00 & 1.00 \\
0.1 & 0.21 & 0.28 & & 1.11 & 1.06 & 1.07 \\
0.2 & 0.17 & 0.32 & & 1.22 & 1.12 & 1.15 \\
0.3 & 0.12 & 0.36 & & 1.37 & 1.20 & 1.25 \\
0.4 & 0.08 & 0.41 & & 1.56 & 1.29 & 1.39 \\
\hline
\end{tabular}

Based on self-consistent calculations of $O^{\prime}$ Connell and Budiansky [1974]. Also shown is the ratio of elastic unloading stiffness of surroundings for undrained conditions to same for drained conditions, for various shapes of the inhomogeneous zone.

teristic diffusion time $t_{D}\left(=a^{2} / c\right)$ to the time for the remote tectonic strain to increase by amount $\lambda$, where $\lambda$ is defined in Figure 6.

Some specific plots of the results represented by (34) will be shown subsequently. First we discuss the choice of parameters. We have examined the cases $\xi=1$ and $\xi=10$. The first corresponds to a near-spherical inhomogeneous zone (e.g., (3) yields $\xi=1$ when a drained Poisson ratio $\nu=0.2$ is assumed). The second corresponds to a flattened, slitlike zone; from (4), $\xi$ $=10$ is consistent with an axisymmetric ellipsoid with aspect ratio $a / b \approx 18$. Remarkably, our numerical results indicate that for given values of $R$ and $\xi / \xi_{u}$ the function $g$ of (34) is very nearly independent of $\xi$. Results for the time $\theta$ to go from point $B^{\prime}$ to point $D^{\prime}$ in Figure 4, for example, typically differ by $1 \%$ or less for $\xi=1$ versus $\xi=10$, except at the largest values of $R$ that we considered $(R \approx 3)$, at which the differences were still only of the order of $10 \%$.

The ratio $\xi / \xi_{u}$ in (34) can be interpreted via (2) as the ratio of the elastic unloading stiffness of the inclusion surroundings under undrained conditions to the same under drained conditions. From (3), (4), and (5) the ratio is given by

$$
\begin{gathered}
\frac{\xi}{\xi_{u}}=\frac{(4-5 \nu)\left(7-5 \nu_{u}\right)}{\left(4-5 \nu_{u}\right)(7-5 \nu)} \\
\frac{\xi}{\xi_{u}}=\frac{(1-\nu)\left(2-\nu_{u}\right)}{\left(1-\nu_{u}\right)(2-\nu)} \quad \frac{\xi}{\xi_{u}}=\frac{1-\nu}{1-\nu_{u}}
\end{gathered}
$$

for the cases of spherical, narrow axisymmetric ellipsoidal, and narrow elliptical inclusions, respectively. The ratio evidently depends on $\nu$ and $\nu_{u}$, and there is no direct source of in situ values known to us. Neither corresponds to the Poisson ratio inferred from seismic wave speed ratios, as noted by Rice and Cleary [1976] and O'Connell and Budiansky [1977]. Further, in situ values will almost certainly be dominated by the presence of joints and fractures and will thus differ significantly from values inferred for intact laboratory specimens. For example, Rice and Cleary [1976] summarize data on intact specimens and report values of $\nu$ and $\nu_{u}$ of 0.27 and 0.30 for Charcoal granite, 0.25 and 0.34 for Westerly granite, 0.12 and 0.31 for Ruhr sandstone, and 0.20 and 0.33 for Berea sandstone. Large, partially opened joints or fractures reduce the drained volumetric stiffness of rock as much as a far larger volume fraction of equiaxed pores, yet they have almost no effect on the undrained volumetric stiffness, assuming full saturation of the flat pore space by liquid water. The effect is to reduce $\nu$ and increase $\nu_{u}$ so that the presence of such joints could make the $\nu$ and $\nu_{u}$ values for a granite rock resemble much more closely the values for intact, porous sandstones than the values for intact granites.

An alternate approach to the effects of joints and fractures is to use the theoretical estimates of elastic properties of cracked rocks by $O^{\prime}$ Connell and Budiansky [1974]. What those authors refer to as the Poisson ratio $\bar{\nu}$ for dry conditions corresponds to our drained ratio $\nu$, and in Table 3 we summarize their results for $\nu$ as a function of their crack density parameter $N r^{3}(=\epsilon)$ for a rock having a Poisson ratio of 0.25 when crack free. Here $N$ is the number of penny-shaped cracks per unit volume having radius $r$. The undrained Poisson ratio $\nu_{u}$ can be calculated from the values of the dry shear modulus $G$ reported by $O^{\prime}$ Connell and Budiansky [1974] and from the undrained bulk modulus $K_{u}$ which, as we have noted, is essentially equal to the bulk modulus of the solid for flat cracklike pore spaces. The resulting values of $\nu_{u}$ are also listed in Table 3. We note that for crack densities $N r^{3}$ greater than approximately 0.1 the crack interactions considered by $O^{\prime}$ Connell and Budiansky lead to results for $\nu$ and $\nu_{u}$ that differ substantially from estimates made on the basis of dilute concentration formulae for $G$ and $K$ [Rice, 1977a].

With the results in Table 3 we can calculate the undrained to drained stiffness ratios $\xi / \xi_{u}$ of the surroundings from (36), and the results are shown in the table for different shapes of the inhomogeneous zone. Now, from observations on wave speed

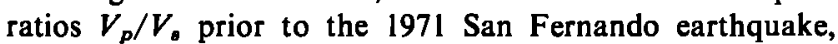
$O^{\prime}$ Connell and Budiansky [1974] suggest that their crack density parameter should have a range from 0.1 to 0.3 to fit one set of seismic data and from 0.2 to 0.4 to fit another. On perusing Table 3, this suggests that stiffness ratios $\xi / \xi_{u}=1.10$ and 1.25 might be taken as being representative, and we have used these two ratios in our numerical evaluations of the function in (34).

\section{Results of Numerical Solutions and Precursory Predictions}

As will be seen, a value of the tectonic loading rate parameter $R=10^{-2}$ is representative of the middle of the range considered in some subsequent numerical evaluations, and we show in Figures 10 and 11 the solution of the integral equation (33) for stiffness ratios of 1.25 and 1.10 , respectively. The points marked $B^{\prime}$ correspond to the drained instability point of Figure 4 beyond which the system is self-driving, and those marked $D^{\prime}$ to the undrained instability point at which dynamic failure occurs. The lower graphs in Figures 10 and 11 have an enlarged time scale and show details in the neighborhood of the instability. For comparison the dashed lines curves show the corresponding progression to instability when pore fluid 

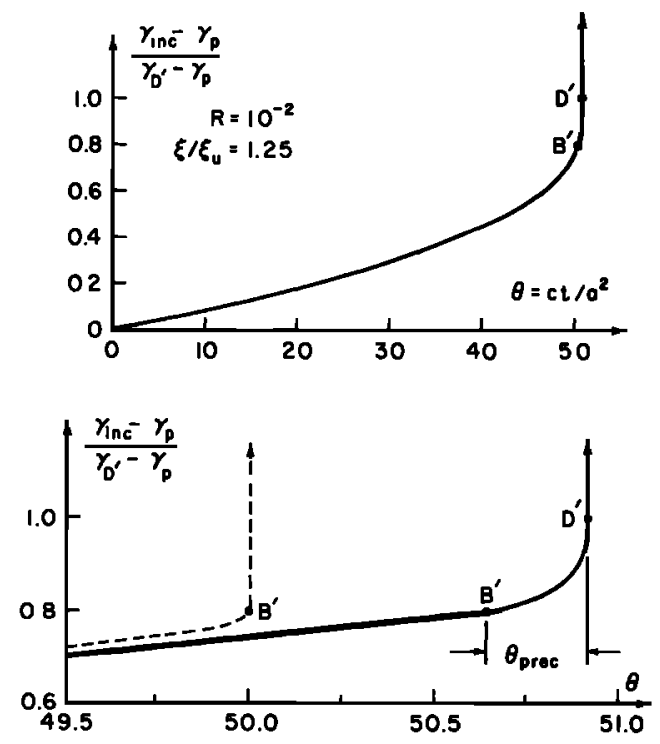

Fig. 10. Postpeak strain history for constant tectonic loading rate, based on undrained to drained stiffness ratio of 1.25. Dashed curve neglects pore fluid effects. Points $B^{\prime}$ and $D^{\prime}$ correspond to those in Figure 4.

effects are neglected (this corresponds to the construction in Figure $2 b$, and the details are explained in Appendix 2).

The effects of the pore fluid are evident in leading to the more prolonged period of accelerating strain before the instability, and, of course, the effect is more pronounced for the larger stiffness ratio (Figure 10) than for the smaller (Figure 11). The choice of a time interval which could be defined as a precursory period is, of course, somewhat arbitrary. As with our dilatant hardening analysis, we define

$$
\theta_{\text {prec }}=c t_{\text {prec }} / a^{2}=t_{\text {prec }} / t_{D}
$$

as the time for traversal of the self-driven range between points $B^{\prime}$ and $D^{\prime}$, and this time interval is shown as a function of $R$ in Figure 12. It is, evidently, a period over which local strains in the zone that is soon to be ruptured accelerate significantly over those accumulated in previous periods of comparable
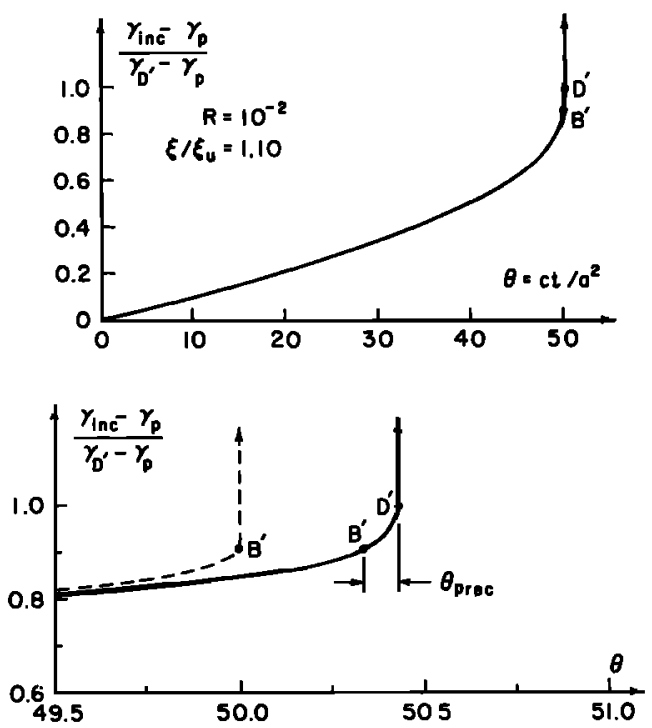

Fig. 11. Same as Figure 10 but for undrained to drained stiffness ratio of 1.10 .

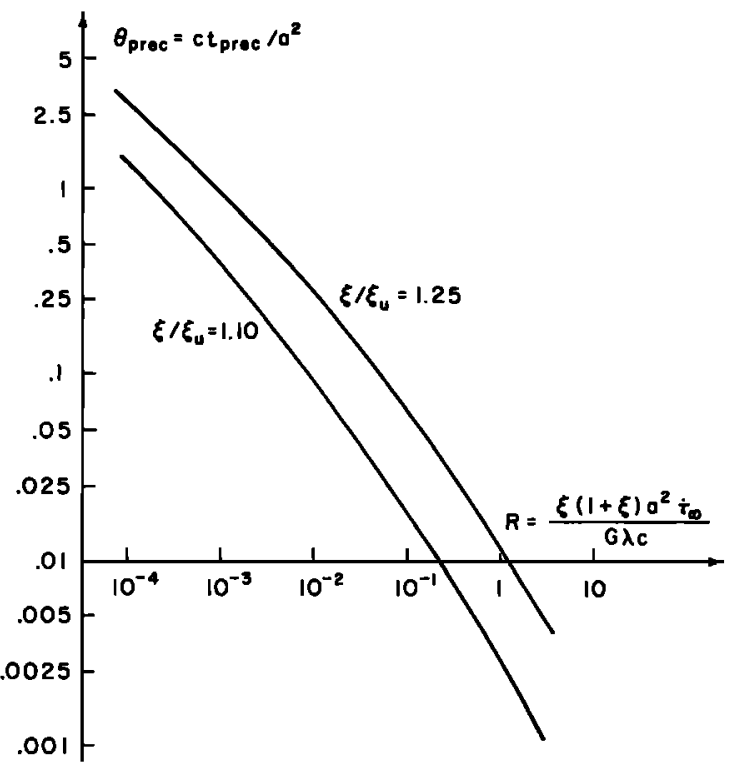

Fig. 12. Precursory times as function of tectonic loading rate parameter $R$, based on time-dependent relaxation of elastic stiffness of material surrounding inclusion.

duration. Deformations at ground surface vary in proportion to the local strain within the inclusion (which can be regarded as an isolated time-dependent dislocation) and will show a similar time history. Also, the rapid deformations could conceivably lead to discernible variations in seismic and/or transport properties within the failing zone.

Table 4 shows some specific predictions of precursory times in days based on the results in Figure 12. We consider in this table three values, 1,3 , and $5 \mathrm{~km}$, for the radius $a$ of the inclusion; and two inclusion shapes (i.e., values of $\xi$ ), a sphere $(\xi=1)$ and a slitlike ellipsoid $(\xi=10 ; 18: 1$ aspect ratio for axisymmetric geometry). Also, two values of the fluid diffusivity are considered, $c=1$ and $0.1 \mathrm{~m}^{2} / \mathrm{s}$, which appear to be consistent with various field $[D . L$. Anderson and J. H. Whitcomb, 1975] and well head [Kovach et al., 1975] measurements. The first results shown for $t_{\text {prec }}$ are based on a stiffness ratio of 1.10 , and following these we show in parentheses the results for a ratio of 1.25 . In preparing Table 4 it is necessary to associate a value of $R$ (equation (35)) with each value of $\xi, a$, and $c$ corresponding to an entry in the table. This is done by choosing a tectonic stress rate $\dot{\tau}_{\mathrm{o}}=1 \mathrm{bar} / \mathrm{yr}$ and shear modulus $G=200$ kbar as previously. Evidently, from Figure 12 a tenfold reduction in $\dot{\tau}_{\infty}$ would lengthen all precursory times shown by a factor of 5 or so. The parameter $\lambda$ of (35) is defined with reference to the stress-strain curve of Figure 6, and, as remarked earlier, if the curve in Figure 6 is to have a peak strength of $1 \mathrm{kbar}$ and to be linear up to of peak strength, with continuous slope where the linear portion joins the parabola, then $\lambda=0.0025$. We use this value in the table.

The results in Table 4 reveal that while $t_{\text {prec }}$ increases with inclusion size, it is again not directly proportional to the diffusion time $a^{2} / c$. Indeed, a tenfold decrease in diffusivity increases $t_{\text {prec }}$ by a factor of 2-3, and a fivefold increase in inclusion size (hence 25 -fold in $a^{2}$ ) increases $t_{\text {prec }}$ by a factor ranging from approximately 2 to 4 .

There is a significant effect of the shape of the inhomogeneous zone. Assuming, as we have, the same $\tau_{\text {Ine }}$ versus $\gamma_{\text {Inc }}$ relation in each case, the spherical zone has precursory times that are 10-20 times longer than those for the flat zone 
TABLE 4. Predicted Precursor Times Based on Time-Dependent Elastic Stiffness of Surroundings

\begin{tabular}{|c|c|c|c|}
\hline Zone Shape & $a=1 \mathrm{~km}$ & $a=3 \mathrm{~km}$ & $a=5 \mathrm{~km}$ \\
\hline Spherical $(\xi=1)$ & $\begin{aligned} R & =1.27 \times 10^{-4} \\
t_{\mathrm{prec}} & =14(29)\end{aligned}$ & $\begin{aligned} m^{2} / s & \\
R & =1.14 \times 10^{-9} \\
t_{\text {prec }} & =36(92)\end{aligned}$ & $\begin{aligned} R & =3.16 \times 10^{-3} \\
t_{\mathrm{prec}} & =55(150)\end{aligned}$ \\
\hline Flat $(\xi=10)$ & $\begin{aligned} R & =6.96 \times 10^{-3} \\
t_{\text {prec }} & =1.3(3.9)\end{aligned}$ & $\begin{aligned} R & =6.27 \times 10^{-2} \\
t_{\text {prec }} & =2.6(8.9)\end{aligned}$ & $\begin{aligned} R & =1.74 \times 10^{-1} \\
t_{\text {prec }} & =3.5(12)\end{aligned}$ \\
\hline Spherical $(\xi=1)$ & $\begin{aligned} R & =1.27 \times 10^{-\mathrm{s}} \\
t_{\mathrm{prec}} & =37(96)\end{aligned}$ & $\begin{aligned} m^{2} / s & \\
R & =1.14 \times 10^{-2} \\
t_{\text {prec }} & =83(261)\end{aligned}$ & $\begin{aligned} R & =3.16 \times 10^{-2} \\
t_{\text {prec }} & =122(399)\end{aligned}$ \\
\hline Flat $(\xi=10)$ & $\begin{aligned} R & =6.96 \times 10^{-2} \\
t_{\text {prec }} & =2.8(9.5)\end{aligned}$ & $\begin{aligned} R & =6.27 \times 10^{-1} \\
t_{\mathrm{prec}} & =4.5(18)\end{aligned}$ & $\begin{aligned} R & =1.74 \\
t_{\mathrm{prec}} & =5.2(22)\end{aligned}$ \\
\hline \multicolumn{4}{|c|}{ Scholz et al. [1973] } \\
\hline
\end{tabular}

Values of $t_{\text {prec }}$ and $t_{D}$ are in days. Precursor times are for the transition from point $B^{\prime}$ to point $D^{\prime}$ in Figure 4. First values shown are for undrained/drained stiffness ratio, $\xi / \xi_{u}$, of 1.10 ; values which follow in parentheses are for ratio of 1.25 . Other data used: $\dot{\tau}_{\mathrm{o}}=1 \mathrm{bar} / \mathrm{yr}, G=200 \mathrm{kbar}$, peak strain parameter $\lambda$ (Figure 6$)=0.0025$. Flat zone corresponds to axisymmetric ellipsoid with $18: 1$ aspect ratio. For $c=1 \mathrm{~m}^{2} / \mathrm{s}, t_{D}=11.6,104$, and 289 days for $a=1,3$, and $5 \mathrm{~km}$, respectively. For $c=0.1 \mathrm{~m}^{2} / \mathrm{s}, t_{D}=116$, 1042 , and 2894 days for $a=1,3$, and $5 \mathrm{~km}$, respectively.

considered. There is also a significant dependence on the stiffness ratio; $t_{\text {prec }}$ for $\xi / \xi_{u}=1.25$ is $2-4$ times longer than it is for $\xi / \xi_{u}=1.10$.

Comparing Tables 1 and 4 , it is seen that $t_{\text {prec }}$ for spherical zones due to dilatant hardening is $2-5$ times longer than the mean (for the two $\xi / \xi_{u}$ ratios) of that due to time-dependent stiffness. Of course, if smaller values of the dilatancy factor $\beta$ are considered, as in Table 2, the $t_{\text {prec }}$ values become more nearly comparable. We have commented that $t_{\text {prec }}$ due to dilatancy should be shorter for flat zones than for spherical zones, but there are no comparisons to be made between the two mechanisms for flat zones.

It is interesting that the $t_{\text {prec }}$ values predicted by solving the integral equation (33) are not very different from those estimated by Rice [1977a] on the basis of an approximation to the response function $f(\theta)$ of Figure 5. Rice's procedure amounts to replacing $f(\theta)$ by a simple exponential form appropriate to a 'standard linear model' with relaxation time $t_{D} / 10$. This has the effect of converting (33) to a first-order nonlinear differential equation. The resulting approximation to $f(\theta)$ is not very close, but predicted $t_{\text {prec }}$ values agree within typically $25 \%$ or so with the more exact results in Table 4 . The cost associated with solving (33) is much larger than it is for solutions based on the standard linear model, mainly because of the necessity of computing error functions in the complex plane to determine $f(\theta)$ in (33); see Appendix 2. Hence in future calculations of the kind reported here, it may suffice to use the approximation based on the standard linear model.

\section{Concluding Discussion}

We have demonstrated that the coupling of pore fluid diffusion with deformation can delay the onset of rapid failure and give rise to precursory periods of quasi-static but accelerating deformation. Our calculations for the duration of this precursory period indicate that the effects can be significant for values of the parameters which are consistent with existing experimental and observational data. More specifically, Figures 8-11 demonstrate that the precursory effects are much more dramatic for fluid-infiltrated solids than they are for those for which the pore fluid is absent. Further, we have presented results for the precursory time so that its dependence on constitutive properties will be evident; see Figure 7 and Tables 1 and 2 for the dilatant hardening mechanism and Figure 12 and Table 4 for the mechanism based on timedependent elastic stifiness. Tables 1 and 4 are based on a range of material properties which we think may be appropriate to crustal rocks at depths of the order of $5 \mathrm{~km}$. The consequences of other choices for the constitutive parameters and loading rate may be estimated from Table 2 and Figures 7 and 12 .

Because much of the discussion of precursory pore fluid effects has concerned their role in connection with the possibility that dilatancy may cause alterations in seismic wave speeds, we emphasized that the effects described here can be important even if conditions are not suitable for wave speed alterations. Indeed, the mechanism of time-dependent stiffness of the surroundings is not contingent upon the dilatant inelastic opening of pores or cracks. Moreover, our calculations for the dilatant hardening mechanism suggest that an amount of dilatancy much smaller than that necessary to affect wave speeds can have a considerable effect of the kind described here in stabilizing the rupture process and that the magnitude of the pore pressure decrease induced by dilatancy alone becomes large only in close proximity to instability. For example, reductions of seismic wave speeds are sometimes postulated to result from the opening of vapor-filled or dry crack space in extensive regions of rock, and this process would be accompanied by large suctions in the pore fluid. But results such as those in Figures 8 and 9 suggest that large suctions can result only very late in what we identify as the precursory period, and it is not clear as to whether they will typically be large enough to alter wave speeds significantly. Further, there is nothing in our analysis which suggests a source of the return that is sometimes suggested of seismic properties to normal levels just before rupture. These conclusions could, however, be a consequence of oversimplifications in our model. For example, the weakening zone is regarded as being spatially uniform up to dynamic instability, and no provision is made for a gradual concentration of deformations into a narrow fault zone.

Because of the uncertainty of precursor time estimates based on observations prior to earthquakes, it is difficult to draw definitive conclusions by comparison of our predicted pre- 
cursor times with observations. In addition, while our definition of precursor time is unambiguous within the context of our model, it is likely to be an upper limit for precursory times as detected by surface observations. This is because the strain within the inclusion continuously accelerates (Figures 8-11), and it may only be toward the latter portion of the precursory period that effects are significant enough to be observed at ground surface. Of course, strain and tilt at the surface will have a time history similar to that of the inclusion strain, although the magnitude will be attenuated approximately in proportion to the inverse square of distance from the source. In addition, the accelerating strain near failure may also have a discernible effect on transport properties in the source region, for example, on electrical resistivity due to the progressive microfracturing that accompanies inelastic straining. We note that transport properties seem likely to be more affected than seismic properties, at least to the extent that for significant alterations the latter require suctions that are large enough to deplete pore spaces of liquid.

To establish minimum estimates of the precursory time interval, we adopt the more conservative estimates of the size of constitutive parameters expressive of coupling between the rock and its pore fluid. Then it would appear that precursory times would be in the neighborhood of 10-50 days for an approximately spherical weakening zone of $1-\mathrm{km}$ radius. For example, if we modify the entries in Table 1 by use of the lowest dilatancy factor in Table $2(\beta=0.075$, one quarter of the value that we suggest as being representative of laboratory triaxial tests on coherent rock), we obtain $t_{\text {prec }} \approx 11-47$ days for a range of fluid diffusivities between 1 and $0.1 \mathrm{~m}^{2} / \mathrm{s}$. Similarly, from Table 4 and using the smaller ratio of undrained to drained elastic stiffness, $\xi / \xi_{u}=1.10$, we find $t_{\text {prec }} \approx 14-37$ days for the same range of diffusivities. If, for example, the last fifth of the precursory time interval is regarded as being 'readily' detectable, in view of the rapid acceleration of strain near instability, this minimal estimate of the precursory period for a spherical zone of $1-\mathrm{km}$ radius is in the range of approximately 2-10 days. We would suggest this sort of period as being most appropriate in searching for precursory effects of the kind we describe.

Larger spherical zones lead to longer precursory periods, but the predicted effect does not scale directly with the characteristic diffusion time, $t_{D}=a^{2} / c$. Instead, a much less rapid variation occurs, more nearly proportional to $a$ to the power 1 or lower, although no single power can fit all the size dependencies documented in Tables 1 and 4.

An analysis of the dilatant hardening mechanism has not been possible for the nonspherically shaped zones, although an approximate analysis of the time-dependent stiffness mechanism has been possible for ellipsoidal zones. The results in Table 4 correspond to a flattened axisymmetric ellipsoid of approximately 18:1 aspect ratio, and in this case the precursory times are reduced by a factor of approximately 10 . The results for other aspect ratios can be obtained from Figure 12, using the appropriate value of $\xi$. When precursory deformations are concentrated from the start on a single fault plane, it is probably inappropriate to model the process by a narrow inclusion as in the present work. Instead, a model like that suggested in Figure $1 b$ seems more appropriate. This involves the spread of a slipping region along an existing fault under the driving force of the stress concentrations at the edges of the slipping region. We leave the fuller analysis of this case to subsequent work.

The model which we have employed here is of course idealized, and we have made many simplifications. We have consid- ered separately each of the two stabilizing mechanisms of the pore fluid, but it seems evident that the effects will be more pronounced when both act together. For a given $\tau_{\text {inc }}$ versus $\gamma_{\text {inc }}$ curve, undrained runaway generally occurs at a larger strain for the dilatant hardening mechanism than for that of time-dependent stiffness, although the strains at final instability are comparable for smaller values of the dilatancy factor $\beta$ or the modulus $K_{f}^{\prime}$ (see (28)). Although we have considered only parabolic $\tau_{\text {inc }}$ versus $\gamma_{\text {inc }}$ curves, the computations do not appear to be strongly sensitive to their shape as expressed by $\lambda$. Nevertheless, the form which we adopted has a continuously decreasing slope so that dynamic rupture is inevitable. There does, however, exist the possibility that the inclusion stressstrain relation reverses curvature before point $D^{\prime}$ is reached in Figures 3 and 4 (e.g., see Figure $2 c$ ). In such cases it is possible that the pore fluid effects allow the inclusion to undergo strain in the form of a wholly stable creep episode.

More generally, we have shown that if fissured rock masses are fluid infiltrated, then the coupling of the deformation with the diffusion of pore fluid will be important in processes preparatory to faulting. As we remarked earlier, consideration of pore fluid effects has been primarily limited to aspects which we would regard as secondary to their role as setting the time scale of the failure process. We would argue that pore fluid stabilization of faulting merits more attention, but at the same time we stress that these processes are sensitive to values of material parameters and transport properties and there is a need for better data in this regard.

\section{APPENDIX 1}

Equations (22) and (30) were solved numerically for $p(t)$ and $\gamma_{\text {inc }}(t)$ by discretizing the convolution integral in (30). We first write the convolution integral as a sum of integrals over each time step $\Delta \theta=\theta / n$ :

$$
\begin{aligned}
I=\int_{0}^{\theta} \frac{1}{\left[\pi\left(\theta-\theta^{\prime}\right)\right]^{1 / 2} d \theta} & \left(\theta^{\prime}\right) d \theta^{\prime} \\
& =\sum_{k=1}^{n} \int_{(k-1) \Delta \theta}^{k \Delta \theta} \frac{1}{\left[\pi\left(n \Delta \theta-\theta^{\prime}\right)\right]^{1 / 2} d \theta}\left(\theta^{\prime}\right) d \theta^{\prime}
\end{aligned}
$$

where $\theta=t / t_{D}$. For each integral, $d p / d \theta$ was assumed to vary linearly between its values at the limits. The remaining integrations can be performed analytically, and the result may be rearranged to yield

$$
I \approx(4 / 3)(\Delta \theta / \pi)^{1 / 2}\left(\dot{p}_{n}+b_{n} \dot{p}_{0}+\sum_{k=1}^{n-1} K_{k} \dot{p}_{n-k}\right)
$$

where

$$
\begin{aligned}
\dot{p}_{k} & =\frac{d p}{d \theta}(k \Delta \theta) \\
b_{n} & =(3 / 2) n^{1 / 2}-n^{3 / 2}+(n-1)^{3 / 2} \\
K_{k} & =(k+1)^{3 / 2}-2 k^{3 / 2}+(k-1)^{3 / 2}
\end{aligned}
$$

Using (A1) in (30) yields

$$
\begin{aligned}
\dot{p}_{n}= & -\left\{\beta K_{f}^{\prime} G \dot{\gamma}_{\infty}+\left(h-h_{d}\right)\right. \\
& \left.\cdot\left[p_{n}+\frac{4}{3}\left(\frac{\Delta \theta}{\pi}\right)^{1 / 2}\left(b_{n} \dot{p}_{0}+\sum_{k=1}^{n-1} K_{k} \dot{p}_{n-k}\right)\right]\right\} \\
& \cdot\left[h-h_{u}+A\left(h-h_{d}\right) \frac{4}{3}\left(\frac{\Delta \theta}{\pi}\right)^{1 / 2}\right]-1
\end{aligned}
$$


where

$$
A=\frac{3 K_{f}^{\prime}}{\alpha\left(1+4 G / 3 K_{s}\right) N}
$$

Standard fourth-order Runge-Kutta integration formulae were used to solve (A2) and (22).

It is clear from the form of (A2) and (27), and from the asymptotic analysis of Rudnicki [1977b] that $\dot{\gamma}_{i p c}$ and $\dot{p}$ become unbounded as the point $D^{\prime}$ in Figure 3 is approached. Consequently, the numerical calculation must be truncated before $h=h_{u}$. Nevertheless, the contribution to the precursor time which was omitted by the truncation was negligible within the accuracy needed, and no special effort was made to resolve the final instability. Typical examples are shown in Figures 8 and 9, where, although the calculation was truncated well before the strain had reached its value at final runaway, the inclusion obviously would have reached this strain in an extremely short amount of additional time, at least as measured by $\theta$.

\section{APPENDIX 2}

To solve the integral equation (33) subject to the stressstrain relation (31), we introduce the notations

$$
g=\left(\gamma_{\text {inc }}-\gamma_{p}\right) /\left(\gamma_{D^{\prime}}-\gamma_{p}\right)
$$

as in (34), noting that $\gamma_{D^{\prime}}-\gamma_{p}=\lambda / \xi_{u}$ and $\alpha=\xi / \xi_{u}$. Then (33) becomes

$$
\begin{gathered}
g(\theta)-g^{2}(\theta) / 2=[(\alpha+\xi) /(1+\xi)] R \theta / \alpha^{2}+(\alpha(\alpha-1) \\
\cdot \int_{0}^{\theta} f\left(\theta-\theta^{\prime}\right)\left[\xi R / \alpha^{2}(1+\xi)+g\left(\theta^{\prime}\right) d g\left(\theta^{\prime}\right) / d \theta^{\prime}\right] d \theta^{\prime}
\end{gathered}
$$

From Rice et al. [1977],

$$
f(\theta)=1+2[3 \eta(4-3 \eta)]^{-1 / 2} \operatorname{Im}\left[\beta \exp \left(\beta^{2} \theta\right) \operatorname{erfc}\left(\beta \theta^{1 / 2}\right)\right]
$$

where $2 \beta=3 \eta-i[3 \eta(4-3 \eta)]^{1 / 2}$ and Im means 'imaginary part of.' The function is shown in Figure 5 and is not very sensitive to $\eta$ over the allowable range; we use $\eta=0.9$ in our calculations.

The time interval in (A3) is divided into a series of steps. A small initial step is assumed, and each new step is chosen so that the increment in $g$, predicted from the last calculated value of $d g / d \theta$, is smaller than some limiting size chosen to guarantee numerical convergence (the necessary step sizes decrease in approximate proportion to the size of $R$ ). The right side of (A3) is evaluated by assuming that $d g / d \theta$ is constant $(\Delta g / \Delta \theta)$ in each step and by treating $f\left(\theta-\theta^{\prime}\right)$ as constant in each step, using the midstep value of $\theta^{\prime}$. Consequently, at the end of each step (A3) becomes a quadratic equation which is solved for $g(\theta)$ so that calculations for a new step can begin. The results for $R=10^{-2}$ are shown as the solid curves for $g$ in Figures 10 and 11 , and the resulting precursory times are shown in Figure 12 for a wide range of $R$ values. As remarked, for given values of $R$ and $\alpha$, there are negligible differences between results for $\xi=1$ and 10 .

The dashed curves of Figures 10 and 11 correspond to neglecting pore fluid effects on stiffness and are equivalent to replacing $f$ by its long-time value of unity in (A3). Thus by elementary calculations one shows that the equation of the dashed curves is

$$
\alpha g \equiv \xi\left(\gamma_{\text {inc }}-\gamma_{p}\right) / \lambda=1-(1-2 R \theta)^{1 / 2}
$$

and the inclusion strain rate becomes unbounded at $\theta=1 / 2 R$, corresponding to the attainment of point $B^{\prime}$ in Figures 2 and 4. This shows that $R$ may additionally be interpreted as half the ratio of the diffusion time $t_{D}$ to the time for remote loadings to bring the inclusion strain from that at peak strength to that at instability.

Acknowledgments. This research was supported by the U.S. Geological Survey Earthquake Hazards Reduction Program through contract 14-08-0001-15866 with Brown University and contract 14-080001-16795 with the California Institute of Technology and by the National Science Foundation Geophysics Program through grant EAR 74-13697 A01 to Brown University. We are grateful to V. C. Li for programing the numerical calculations outlined in Appendix 2 and that led to Figures 10-12.

\section{REFERENCES}

Anderson, D. L., and J. H. Whitcomb, Time dependent seismology, J. Geophys. Res., 80, 1497-1503, 1975.

Anderson, O. L., and P. C. Grew, Stress corrosion theory of crack propagation with applications to geophysics, Rev. Geophys. Space Phys., 15, 77-104, 1977.

Biot, M. A., General theory of three dimensional consolidation, $J$. Appl. Phys., 12, 155-164, 1941.

Biot, M. A., Nonlinear and semilinear rheology of porous solids, $J$. Geophys. Res., 78, 4924-4937, 1973.

Booker, J. R., Time dependent strain following faulting of a porous medium, J. Geophys. Res., 79, 2037-2044, 1974.

Brace, W. F., and R. J. Martin, A test of the law of effective stress for crystalline rocks of low porosity, Int. J. Rock Mech. Mining Sci., 5, 415-436, 1968.

Brace, W. F., B. W. Paulding, Jr., and C. H. Scholz, Dilatancy in the fracture of crystalline rocks, J. Geophys. Res., 7I, 3939-3953, 1966.

Cleary, M. P., Heterogeneity and porosity effects on the response of geomaterials, in Advances in Civil Engineering Through Engineering Mechanics, pp. 360-363, American Society of Civil Engineers, New York, 1977.

Crouch, S. L., Experimental determination of volumetric strains in failed rock, Int. J. Rock Mech. Mining Sci., 7, 589-603, 1970.

Dieterich, J. H., Time-dependent friction in rocks, J. Geophys. Res., 77, 3690-3697, 1972.

Dieterich, J. H., Time-dependent friction and mechanics of stick-slip, Pure and Appl. Geophys., in press, 1978.

Eshelby, J. D., The determination of the elastic field of an ellipsoidal inclusion and related problems, Proc. Roy. Soc., Ser. A, 24l, 376396, 1957.

Frank, F. C., On dilatancy in relation to seismic sources, Rev. Geophys. Space Phys., 3, 485-503, 1965.

Jaeger, J. C., and N. G. W. Cook, Fundamentals of Rock Mechanics, 2nd ed., Methuen, London, 1976.

King, C.-Y., R. D. Nason, and D. Tocher, Kinematics of fault creep, Phil. Trans. Roy. Soc. London, Ser. A, 274, 355-360, 1973.

Kovach, R. L., A. Nur, R. L. Wesson, and R. Robinson, Water-level fluctuations and earthquakes on the San Andreas fault zone, Geology, 3. 437-440, 1975.

Martin, R. J., Time-dependent crack growth in quartz and its application to creep in rocks, J. Geophys. Res., 77, 1406-1419, 1972.

Martin, R. J., and W. B. Durham, Mechanisms of crack growth in quartz, J. Geophys. Res., 80, 287-293, 1975.

Nur, A., Dilatancy, pore fluids, and premonitory variations of $t_{8} / t_{p}$ travel times, Bull. Seismol. Soc. Amer., 78, 1217-1222, 1972.

Nur, A., and J. D. Byerlee, An exact effective stress law for elastic deformation of rock with fluids, J. Geophys. Res., 76, 6414-6419, 1971.

O'Connell, R. J., and B. Budiansky, Seismic velocities in dry and saturated cracked solids, J. Geophys. Res., 79, 5412-5426, 1974.

O'Connell, R. J., and B. Budiansky, Viscoelastic properties of fluid saturated cracked solids, J. Geophys. Res., 82, 5719-5735, 1977.

Prescott, W. H., and J. C. Savage, Strain accumulation on the San Andreas fault near Palmdale, California, J. Geophys. Res., 81, 4901$4908,1976$.

Reynolds, O., On the dilatancy of media composed of rigid particles in contact, with experimental illustrations, Phil. Mag., 1885. (Reprinted in Papers on Mechanical and Physical Subjects by O. Reynolds, vol. 2, pp. 203-216, Cambridge University Press, New York, 1901.) 
Rice, J. R., On the stability of dilatant hardening for saturated rock masses, J. Geophys. Res., 80, 1531-1536, 1975.

Rice, J. R., Theory of precursory processes in the inception of earthquake rupture, in Proceedings of the Symposium on Physics of Earthquake Sources, German Democratic Republic (East German) Academy of Sciences, Berlin, 1977a.

Rice, J. R., Pore pressure effects in inelastic constitutive formulations for fissured rock masses, in Advances in Civil Engineering Through Engineering Mechanics, pp. 360-363, American Society of Civil Engineers, New York, $1977 b$.

Rice, J. R., and M. P. Cleary, Some basic stress diffusion solutions for fluid-saturated elastic porous media with compressible constituents, Rev. Geophys. Space Phys., 14, 227-24I, 1976.

Rice, J. R., and D. A. Simons, The stabilization of spreading shear faults by coupled deformation-diffusion effects in fluid-infiltrated porous materials, J. Geophys. Res., 81, 5322-5334, 1976.

Rice, J. R., J. W. Rudnicki, and D. A. Simons, Deformation of spherical cavities and inclusions in fluid-infiltrated elastic solids, Int. J. Solids Struct., 14, 289-303, 1978.

Rudnicki, J. W., The inception of faulting in a rock mass with a weakened zone, J. Geophys. Res., 82, 844-854, 1977 a.

Rudnicki, J. W., Localization of deformation, brittle rock failure, and a model for the inception of earth faulting, Ph.D. thesis, Brown Univ., Providence, R. I., $1977 b$.

Rudnicki, J. W., and J. R. Rice, Conditions for the localization of deformation in pressure-sensitive dilatant materials, J. Mech. Phys. Solids, 23, 371-394, 1975.

Scholz, C. H., Static fatigue of quartz, J. Geophys. Res., 77, 2104$2114,1972$.

Scholz, C. H., and R. Kranz, Notes on dilatancy recovery, J. Geophys. Res., 79, 2132-2135, 1974.

Scholz, C. H., L. R. Sykes, and Y. P. Aggarwal, Earthquake prediçtion: A physical basis, Science, 181, 803-810, 1973.

Swolfs, H., Chemical effects of pore fluids on rock properties, Underground Waste Management and Environmental Implications, $\mathbf{M e m}$. 18, edited by T. D. Cook, pp. 224-234, Amer. Ass. of Petrol. Geol., Tulsa, Okla., 1972.

Zoback, M. D., and J. D. Byerlee, The effect of cyclic differential stress on dilatancy in Westerly granite under uniaxial and triaxial conditions, J. Geophys. Res., 80, 1526-1530, 1975.

(Received March 20, 1978;

revised June 22, 1978;

accepted June 23, 1978.) 